Article

\title{
Bio-Inspired Distributed Transmission Power Control Considering QoS Fairness in Wireless Body Area Sensor Networks
}

\author{
Chan-Jae Lee ${ }^{(1)}$, Ji-Young Jung ${ }^{(1)}$ and Jung-Ryun Lee * \\ School of Electrical and Electronics Engineering, Chung-Ang University, 84 Heukseok-ro, Dongjak-gu, \\ Seoul 06974, Korea; cjleeno22@cau.ac.kr (C.-J.L.); jiyoung@cau.ac.kr (J.-Y.J.) \\ * Correspondence: jrlee@cau.ac.kr; Tel.: +82-2-820-5820
}

Received: 24 August 2017; Accepted: 11 October 2017; Published: 14 October 2017

\begin{abstract}
Recently, the development of wireless body area sensor network (WBASN) has accelerated due to the rapid development of wireless technology. In the WBASN environment, many WBASNs coexist where communication ranges overlap with each other, resulting in the possibility of interference. Although nodes in a WBASN typically operate at a low power level, to avoid adversely affecting the human body, high transmission rates may be required to support some applications. In addition to this, since many varieties of applications exist in the WBASN environment, each prospective user may have different quality of service (QoS) requirements. Hence, the following issues should be considered in the WBASN environment: (1) interference between adjacent WBASNs, which influences the performance of a specific system, and (2) the degree of satisfaction on the QoS of each user, i.e., the required QoS such as user throughput should be considered to ensure that all users in the network are provided with a fair QoS satisfaction. Thus, in this paper, we propose a transmission power adjustment algorithm that addresses interference problems and guarantees QoS fairness between users. First, we use a new utility function to measure the degree of the satisfaction on the QoS for each user. Then, the transmission power of each sensor node is calculated using the Cucker-Smale model, and the QoS satisfaction of each user is synchronized dispersively. The results of simulations show that the proposed algorithm performs better than existing algorithms, with respect to QoS fairness and energy efficiency.
\end{abstract}

Keywords: Bio-inspired; energy efficiency; fairness; power control; quality of service; wireless body area sensor network

\section{Introduction}

With the rapid advance of wireless technology in recent years, the development of WBASN has been accelerated in an effort to monitor patients and support applications used for telemedicine [1-3]. As the population is growing rapidly around the world, the demand for healthcare systems will consequently increase. Especially the population aged between 60 and 80 is expected to double in 2050, compared to the number in 2010. Thus, technological development in WBASNs is essential. Millions of people die every year from diseases such as cancer and cardiovascular disease, where early detection and treatment can dramatically reduce the mortality rate. Therefore, future healthcare systems should provide preventive services. Wearable devices that can be attached to the body, to monitor and analyze a bio-signal, are typically used as effective precautionary measures, for early disease detection. Many studies have been conducted to address various problems that may occur in the WBASN environment, where these wearable devices are used.

The WBASN can be configured by several wireless technologies that have advantages and disadvantages depending on each radio technology [4]. In this paper, we focus on the IEEE 
802.15.6 standard. A study group on the WBASN communication standard was approved as Task Group 6 (TG6) of the IEEE 802.15 working group, in January 2008. The first draft of the WBASN standard was ratified in February 2012 [5]. The purpose of WBASN communication standardization is "to provide an international standard for a short-range (i.e., about human body range), low power, and highly reliable wireless communication for use in close proximity to, or inside, a human body" [1].

The WBASN has distinctive features compared with the existing wireless networks [6]. In the WBASN environment, there are many WBASN networks where communication ranges overlap with each other. Thus, interference may occur between each WBASN network, because of limited frequency resources [7]. This inter-WBASN interference may cause problems that affect each user, such as a decrease in the throughput, or an increase in the packet loss rate. In general, inter-WBASN interference is maximized when there is no coordination between each network. Generally speaking, techniques for reducing interference can be classified as centralized and distributed methods. With centralized methods, a central coordinator is deployed like a base station, which regulates the media access control (MAC) and transmit power of each sensor node that is dependent on a WBASN, to reduce the effect of interference on each hub. However, since each user in the WBASN environment is highly mobile, and user-dependent WBASNs operate independently of each other, central coordinator based interference mitigation methods are not suitable for deployment with WBASNs. Therefore, distributed methods are used to mitigate interference in the WBASN environment.

Figure 1 shows the transmission power requirements and data rates for different wireless technologies [1]. From this image, it can be seen that the transmit power required in a WBASN environment is significantly lower than with other networks. Since the sensor nodes in the WBASN, for collecting biometric data, may exist on the inside, and on the surface of a human body, lower power consumptions are required for WBASN technology than with existing wireless technologies, in consideration of the specific absorption rate (SAR) of electromagnetic waves. In spite of the limited transmit power of WBASNs, the range of transmission rates required for each application is very broad. This is because the types of data measured by the sensor nodes vary depending on the application, and the required data rate varies depending on the type of data being transmitted. Since a maximum data rate of about $10 \mathrm{Mbps}$ and low power consumption are required in the WBASN environment, the WBASN protocol requires higher energy efficiency than existing protocols. Depending on the type of application being used in a WBASN, the differences in the required data rate may be very large. As a result, some sensor nodes may use an unnecessarily large transmission power, reducing their energy efficiency, as well as the performance of other nodes. In addition, since the types of applications utilized in a WBASN are different, the data rate required for satisfying quality of service (QoS) may also be different for each WBASN. A required transmission rate based on the QoS is directly related to fair treatment of users in the network [8]. If the difference in required data rate for a satisfactory QoS is not taken into consideration, users in the network could be provided with an unfair service. For example, allocating the same amount of resources to a voice service of relatively low quality, and a high-quality video service, does not provide each user with fair treatment. Therefore, in the WBASN environment it is necessary to consider different QoS requirements according to the applications employed in WBASNs.

The remainder of this paper is organized as follows. In Section 2, we present the related works. In Section 3, we describe the general system models and parameters of the WBASN. In Section 4, we explain the Cucker-Smale model, which is the basis of the proposed algorithm. In Section 5, we introduce the operation of the proposed transmission power control algorithm in WBASN. In Section 6, we evaluate the performance of the proposed algorithm. Finally, we conclude this paper in Section 7. 


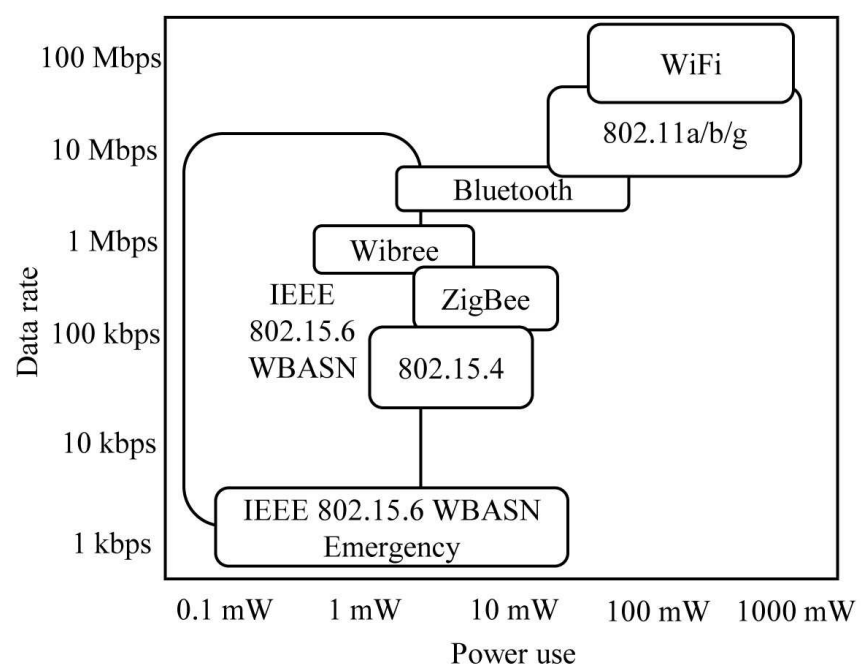

Figure 1. Comparison of power requirements and data rates in different wireless technologies.

\section{Related Works}

The interference problem has been dealt with in the existing network [9]. It is important to design the wireless network by considering the signal-to-interference-ratio (SINR), as indicated by the wide literature available on the topic, which we review in this section. The obtained SINR is proportional to a variety of performance metrics including throughput [10]. However, the frequencies assigned to two connections may incur interference to one another, resulting in quality loss of the signal [11]. For this reasons, several studies in the traditional wireless networks had conducted to mitigate interference. Resource allocation schemes in wireless network have been proposed to assign channel and to associate the users with access points (APs) by considering SINR. To address the co-channel interference problems, a biological behavior-based network resource management method is proposed [12]. A solution to user association with the AP is proposed to guarantee the best quality of service considering SINR [13]. In the cellular networks or the broadcasting systems, the efficiency of SINR is an important factor and the optimization of the SINR problem have been conducted. The planning methodologies in the cellular network that allow to minimize interference overhead while maintaining the established network coverage are introduced [14]. A solution method for SINR constraints problem in the cellular network is proposed that combines combinatorial Benders decomposition, classical Benders decomposition, and valid cuts in a nested way [15]. A two-stage heuristic methodology composed of power and frequency assignment stages is proposed so as to minimize the loss from mutual interference in the broadcasting system [16]. An optimization problem of transmission powers so as to guarantee the required SINR in the broadcasting system can be formulated as a mixed integer linear program, and the analysis on the behavior of this model is proposed [17]. To solve the interference problem caused by the coexistence of a plurality of WBASNs that are dependent on a specific individual, unlike earlier works for the existing wireless networks, many studies considering features of the WBASN have been conducted. The asynchronous inter-network interference avoidance (AIIA) scheme has been proposed, to reduce the magnitude of mutual interference that occurs in situations where two or more WBASNs share the same channel, so that the active interval in each WBASN frame is not allocated at the same time [18]. AIIA is based on a hybrid technique of carrier sense multiple access with collision avoidance (CSMA/CA) and time division multiple access (TDMA). In this scheme, information relating to time occupied in a TDMA interval is periodically exchanged and recorded in the AIIA table. The coordinator in each WBASN can check if its TDMA interval conflicts with the time occupied by a neighbor, based on the information in the AIIA table. If a collision is predicted, the coordinator occupies another time slot. A technique for 
adjusting the modulation parameter, data rate, and duty cycle, linearly, according to the SINR of the receiver has been proposed, to solve co-channel interference [19].

Transmission power control plays an important role in mitigating interference in a wireless network. The coverage extension of base stations (BSs) can be enhanced by mitigating interference in the cellular networks [20]. In this scheme, the optimization of BS parameters leads to improving the system performance. The overall power consumption of AP is also decreased by interference management in the wireless local area network (WLAN) [21]. This approach adapts the branch-and-benders-cut method to solve the non-linear power design problem on the interference management. Many studies have been conducted on methods to improve performance in the WBASN environment by adjusting transmission power. The received signal strength indicator (RSSI) is one of the commonly used parameters for power control algorithm. Due to inter-sensor node interference caused by body movement or variance of channel status, a method for detecting changes to the link quality using the received signal strength indicator (RSSI), and adjusting the transmission power, is proposed [22]. An energy efficient transmission power control scheme in an on-demand way to adapt to varying channel environments is proposed [23]. A power control schemes based on the reinforcement learning (RL) and mathematical optimization are proposed to consider interference. To determine the optimal combination of beamforming and power control in sensor arrays, the RL algorithm is proposed [24]. In this case, the power configuration set of this kind of scheme could be explored by [25]. A method for channel and power allocation based on a RL mechanism and convex optimization is proposed [26]. Studies on game theory-based algorithm have been conducted in existing wireless networks to obtain the optimal transmission power that maximizes system performance. The non-cooperative differential game is used to control transmit power of wireless powered sensor network [27]. This scheme extends working hours and improves throughput by optimal power control. The proactive power update (PAPU) algorithm applied this theory to WBASN environments with the purpose of maximizing the data rate of the entire WBASN networks [7]. The scheme recognizes changes in SINR or transmission power of neighboring WBASNs, and determines whether to adjust the transmission power. If a coordinator decides to adjust transmission power, the optimal power that increases the transmission rate is found using the Nash equilibrium, based on the quality of the channel, interference from other WBASNs, noise, and other predetermined power parameters.

Besides, studies on various problem occurring in WBASN have been conducted. Researches on using relays have conducted to improve performance of far nodes. In the system of using relay node, routing is essential to improve network lifetime and system performance. A global routing scheme using Dijkstra's algorithm is proposed with a novel cost function specialized for balancing energy consumption [28]. A relay selection scheme is proposed to maximize the lifetime of WBASNs through formulating and solving an optimization problem where relay selection of each node acts as optimization variable [29]. Traffic uncertainty causes degradation of network performance and interruption to operate protocols [30]. Therefore, design of network protocol should take into account some uncertain factors in order to make it realistic [31]. In the WBASN, the sensed data is generated by event-driven and this makes the algorithm to be outdated. For this reason, some optimization schemes are proposed to consider the event-driven data generation. A heuristic approach combining deterministic and probabilistic variable fixing strategies is proposed for WBASN optimal design, formulated as an integer programming problem [32]. An original optimization algorithm is proposed that exploits suitable linear relaxations to guide a randomized fixing of the variables [33]. It is supported by an exact large variable neighborhood search. Several robust algorithms considered the SINR. The robust optimization scheme using SINR is proposed to deal with the jamming problem in wireless networks [34]. The stochastic programming-based convex optimization with the probabilistic SINR constraints is proposed to optimize the transmission power [35]. A stochastic revenue optimization model based on bid pricing model for cellular networks is proposed [36]. There also exist techniques that integrate various functions of wireless networks. An integrated optimization is proposed to 
handle the collide functions and to allocate the radio resources [37]. An optimal cross layer design is developed in order to construct hierarchical system [38].

In conventional studies, the objective of transmission power control schemes is to minimize the energy consumption [21], or to maximize the data rate in considering interference occurred in inter-WBASN [7]. Actually, determining the data rate according to QoS requirements in wireless environment is one of the important issues rather than merely considering high transmission rates or energy efficiency [39]. However, as these schemes do not consider the QoS of each user in the WBASN environment, transmission power could operate regardless of the required data rate. Thus, there may be a limit in the ability to provide fair QoS to the users in WBASNs. In this paper, we propose an algorithm that can achieve fair QoS satisfaction across all users in WBASNs considering different QoS levels of the users. First of all, a utility function, which indicates how closely the current data rate matches the required data rate, is defined. Next, based on information from neighboring WBASNs, each network derives the transmission power needed for a QoS satisfaction value equal to the average QoS satisfaction value of the neighboring WBASNs. We use the Cucker-Smale model [40] in calculating the appropriate transmission power for each user. The Cucker-Smale model is typically used to simulate phenomena in which organisms move in groups, based on individual behavior with simple rules, using limited information. The model is thus suitable for the WBASN environment, where the transmission power must be controlled in a distributed manner, because it uses only information available from the neighboring nodes. In addition, the Cucker-Smale model can be applied to solving the problem of fair distribution of a satisfactory QoS in all neighboring WBASNs, because it synchronizes the specific variables of each entity to the same value.

\section{System Model}

In this paper, we employ IEEE 802.15.6-based system as a target system [5]. In IEEE 802.15.6, multiple nodes belong to one coordinator (or hub). The hub shall operate in one of the three access modes: 'beacon mode with superframe', 'non-beacon mode with superframe', and 'non-beacon mode without superframe'. The superframe is divided into contention-based period and managed access period (MAP) as shown in Figure 2. In the contention-based period, there are three types of access phases: exclusive access phase (EAP), random access phase (RAP), and contention access period (CAP). In this period, sensor nodes use CSMA/CA or slotted ALOHA methods to access medium. Otherwise, in the MAP, it is possible to allocate resource by scheduling such as TDMA. If the beacon signal is available, the hub can determine whether to use each access phase or not, and broadcast some information such as the value of the utility function. For this reason, the beacon mode with superframe is used to facilitate a WBASN in our system.

\begin{tabular}{|l|l|l|l|l|l|l|l|l|}
\hline Beacon & EAP1 & RAP1 & MAP & EAP2 & RAP2 & MAP & Beacon2 & CAP \\
\hline
\end{tabular}

Figure 2. The layout of access phase in superframe. EAP, exclusive access phase; RAP, random access phase; MAP, managed access period; CAP, contention access period.

A WBASN can consist of a hub and multiple sensor nodes. Each sensor node has a different QoS requirement, which leads to scheduling issue on resource allocation in the given MAC protocol. In this paper, we assumed that there is no intra-WBASN collision by assuming the TDMA-based WBASN protocol such as IEEE 802.15.6, in order to focus on the performance evaluation of our algorithm proposed in the context of control over inter-WBASN collision. The hub allocates time resources of the MAP to each sensor node by using the beacon signal. Figure 3 shows medium access in the MAP. Each sensor node periodically generates data and transmits it at the allocated time interval. At this time, the sensor node set the Ack field in the MAC header to immediate-Ack (I-Ack). After data transmission 
is completed, the hub sends back I-Ack including the information of calculated transmission power to the sensor node. The sensor node can adjust its power level by using the power information in the I-Ack.

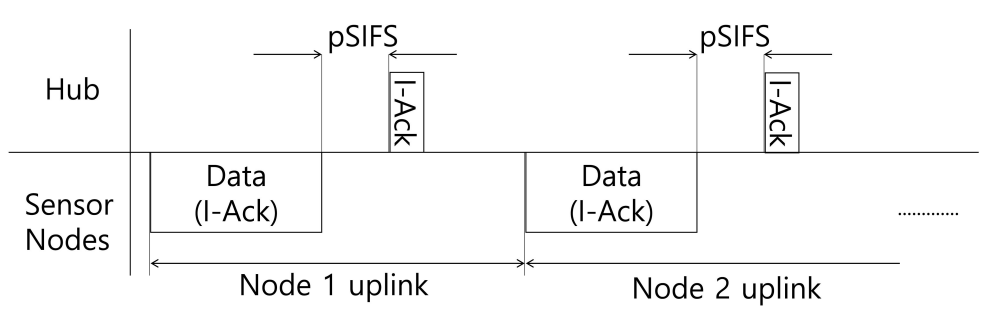

Figure 3. Example of scheduling in MAP. I-Ack, immediate-Ack. pSIFS, Short Inter-Frame Spacing.

It is assumed that a single sensor node $S N_{i}$ exists in $W B A S N_{i}$ because contention-free MAC scheme is assumed to avoid intra-WBASN collision between sensor nodes associated with a WBASN, as shown in many studies on previous power control schemes in the WBASN environment [41-44]. As shown in Figure 4, there may be other WBASNs within transmission range, which may cause interference. Suppose that each user $i$ constitutes a single WBASN WBASN $i$ and one hub $H_{i}$ in $W B A S N_{i}$ collects information from each sensor node. The coefficient $g_{i j}$ represents the channel gain between the transmitter $i$ and the receiver $j$, i.e., The coefficient $g_{i j}$ represents intra-WBASN channel gain in the WBASN when $i=j$, and inter-WBASN interference gain between different WBASNs when $i \neq j$. For example, in Figure 4, $S N_{1}$ tries to transmit measured data to $H_{1}$ in $W B A S N_{1}$, which it belongs to, and, at the same time, $S N_{2}$ tries to transmit data to $H_{2}$ in $W B A S N_{2}$, which causes interference to each other's network.

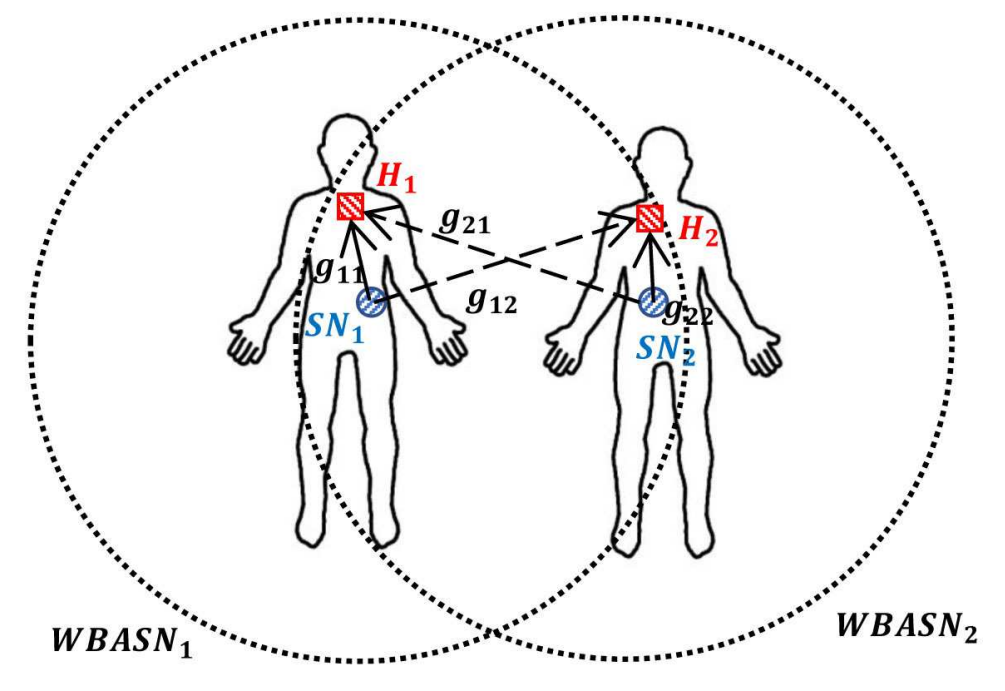

Figure 4. Inter-WBASN interference model.

The SINR, $\gamma_{i}(t)$, of the $i$-th WBASN at time $t$ is:

$$
\gamma_{i}(t)=\frac{p_{i}(t) g_{i i}}{\sum_{\mathbf{j} \in \mathbf{N}_{\mathbf{i}} \mathbf{j} ; \mathbf{j} \mathbf{i}} p_{j}(t) g_{j i}+n_{0}}, \quad i=1,2, \cdots, N
$$


where $p_{i}(t)$ is the transmission power of $S N_{i}, N_{i}$ is the set of neighboring nodes within transmission range of $S N_{i}$, and $n_{0}$ is additive white Gaussian noise (AWGN). According to Shannon's theory, the data rate, $R_{i}(t)$, of the $i$-th WBASN at time $t$ is described as follows:

$$
R_{i}(t)=B \log _{2}\left(1+\gamma_{i}(t)\right), \quad b / s
$$

where $B$ is the bandwidth of the channel. Finally, the energy efficiency, $E_{i}(t)$, of the $i$-th WBASN at time $t$ is defined as,

$$
E_{i}(t)=\frac{R_{i}(t)}{p_{i}(t)}, \quad b / J
$$

\section{Cucker-Smale Model}

When observing naturally occurring phenomena, organisms often move in groups based on individual behavior with simple rules, using limited information. For example, when birds move in groups, they all move at the same speed. Cucker and Smale proposed a model (the Cucker-Smale model) to simulate this phenomenon, using the rule that each object adjusts its speed and direction individually, in consideration of the speed and direction of neighboring objects [40].

This mathematical model deals with the relationship of individuals that interact with each other. Each entity interacts with its neighbors, to adjust its velocity according to the weighted mean of the relative velocities of other neighbors in the group together with its own velocity. Assuming that there are $N$ entities, the position of the $i$-th entity is defined as $x_{i}(t)$, and the velocity is defined as $v_{i}(t)$. Based on the above description, the Cucker-Smale model can be expressed as follows:

$$
\begin{gathered}
\frac{d x_{i}(t)}{d t}=v_{i}(t) \\
v_{i}(t+1)-v_{i}(t)=\frac{\lambda}{N} \sum_{j=1}^{N} \psi\left(\left|x_{j}-x_{i}\right|\right)\left(v_{j}(t)-v_{i}(t)\right)
\end{gathered}
$$

In the above equation, $i=1, \cdots, N, t>0$, and $\lambda$ is a non-negative value indicating the coupling strength between individuals, which also refers to the learning weight. $\psi(\cdot)$ is a function expressing the communication range and weight between the affected entities. A typical $\psi(\cdot)$ function is given as follows:

$$
\begin{gathered}
\psi_{1}\left(\left|x_{j}-x_{i}\right|\right)=1 \\
\psi_{2}\left(\left|x_{j}-x_{i}\right|\right)=1_{\left|x_{j}-x_{i}\right| \leq r} \\
\psi_{3}\left(\left|x_{j}-x_{i}\right|\right)=\frac{1}{\left(1+\left|x_{j}-x_{i}\right|^{2}\right)^{\beta}}
\end{gathered}
$$

In the above equation, $r$ is a positive number, and $\beta$ is a non-negative value. In this paper, we will use Equation (7) to express the communication range between entities. Furthermore, Cucker and Smale demonstrated that the velocities of all individuals converge, when $\psi(\cdot)$ is a non-negative and non-increasing function. Therefore, flocking phenomena satisfy the two following time-asymptotic convergence properties.

$$
\begin{aligned}
& \lim _{t \rightarrow \infty}\left|v_{i}(t)-v_{j}(t)\right|=0, \quad \text { for } \quad i \neq j \\
& \sup _{0 \leq t<\infty}\left|x_{i}(t)-x_{j}(t)\right|<\infty, \quad \text { for } \quad i \neq j
\end{aligned}
$$

Equations (9) and (10) show that the difference in velocity between individuals converges to zero over time, and the distance between individuals does not diverge. 


\section{Proposed Algorithm}

The Cucker-Smale model described in Section 3 is suitable for application to distributed resource allocation, because each node can achieve the global goal by acquiring and processing local information. In the Cucker-Smale model, the velocity vectors of each node are synchronized, as detailed in Equation (9). If the operational principle of the Cucker-Smale model is applied to the synchronization of each WBASN, the data rate of each node can be synchronized. However, different applications exist in the WBASN environment, where the difference in data rate varies, from several tens of kilobits per second, to ten megabits per second. Therefore, QoS fairness cannot be achieved for each node, if they share the same data rate. Moreover, if all users have the same data rate, some users may consume power unnecessarily in maintaining a data rate that is higher than required. To solve these problems, we propose a new power control algorithm for use in the WBASN environment based on the Cucker-Smale model. In the proposed algorithm, termed the flocking-based transmission power control with utility (FTPC-U) algorithm, the QoS of each user is defined as a utility function and the transmission power is adjusted to synchronize the value of the utility. This algorithm also increases the energy efficiency of the network, by preventing unnecessary energy consumption in transmission, and enables each user to be provided with a fair QoS.

\subsection{Utility Function}

In this paper, we define a utility function to express the QoS satisfaction of each user according to the data rate. Existing utility functions used to represent QoS satisfaction typically have a sigmoidal or logarithmic shape [45]. The shapes of these utility functions are applied depending on the type, and characteristics of the applications in use. We express two types of utility as one function. The utility, $U_{i}(t)$, of the $i$-th WBASN at time $t$ is given as follows:

$$
U_{i}(t)=U_{\max }-\frac{e^{-c e^{b \cdot r(t)}}}{e^{-c e^{-b}}-e^{-c}}, \quad r(t)=\frac{R_{i}(t)-R_{i}^{R e q}}{R_{i}^{R e q}}(-1 \leq r(t)<\infty)
$$

where $b$ and $c$ are the control parameters $(b>0, c>0)$, and $R_{i}^{R e q}$ is the required data rate of WBASN $N_{i}$ The shape of the above function can be changed according to the values of control parameters, $b$ and $c$, as shown in Figure 5. The term, $U_{\max }=1+e^{-c} /\left(e^{-c e^{-b}}-e^{-c}\right)$, refers to the maximum possible value of the utility. In this paper, $R_{i}^{R e q}$ does not change over time, but has a fixed value for each user. Since $R_{i}(t)$ has a minimum value of zero, $r(t)$ is greater than or equal to -1 . If $R_{i}(t)$ is zero, the value of $r(t)$ is -1 , and $U_{i}(t)$ is zero. If the values of $R_{i}(t)$ and $R_{i}^{R e q}$ are same, the value of $r(t)$ is zero, and the value of $U_{i}(t)$ is one. $r(t)$ goes to infinity as $R_{i}(t)$ goes to infinity. The value of $U_{i}(t)$ is maximized in this case $\left(U_{i}(t)=U_{\max }\right)$.

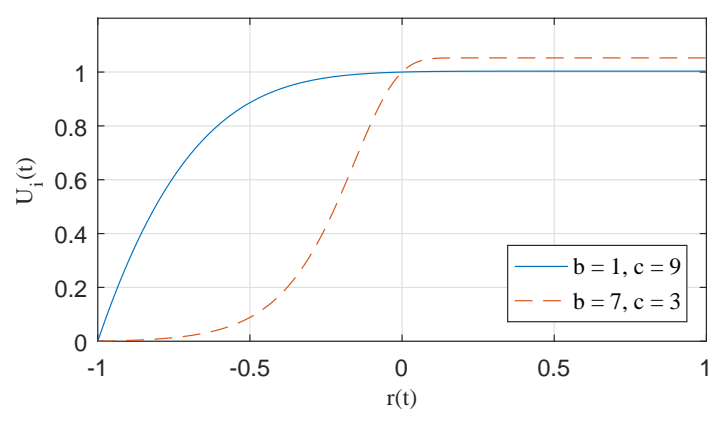

Figure 5. Proposed utility function. 


\subsection{Operation of the FTPC-U algorithm}

We use the above utility function to calculate the utility value of each user, and apply it to the Cucker-Smale model to adjust the transmission power by synchronizing the value of utility function. The operational procedure of the proposed algorithm is as follows:

1. At time $t$, the sensor node $S N_{i}$ of each network $W B A S N_{i}$ transmits a data packet to the hub $H_{i}$ with a transmission power given by $p_{i}(t)$.

2. $H_{i}$ measures the SINR $\gamma_{i}(t)$ based on the received packet.

3. $H_{i}$ computes the current data rate $R_{i}(t)$ using Equation (2).

4. $H_{i}$ calculates the current utility value $U_{i}(t)$ using Equation (11).

5. When the calculated utility is greater than one, i.e., $R_{i}(t)>R_{i}^{R e q}, H_{i}$ reduces the transmission power of $S N_{i}$, such that the current data rate $R_{i}(t)$ becomes the required data rate $R_{i}^{R e q}$. Using the SINR information at time $t, p_{i}^{\text {Req }}(t)$, the transmission power required to convert $R_{i}(t)$ to $R_{i}^{\text {Req }}$, can be obtained from Equations (1) and (2).

$$
\begin{aligned}
R_{i}^{\text {Req }} & =B \log _{2}\left(1+\gamma_{i}^{\text {Req }}(t)\right) \\
& =B \log _{2}\left(1+\frac{p_{i}^{\text {Req }}(t) g_{i i}}{\sum_{\forall j, j \neq i}^{N_{i}} p_{j}(t) g_{j i}+n_{0}}\right) \\
& =B \log _{2}\left(1+p_{i}^{R e q}(t) \times \frac{\gamma_{i}(t)}{p_{i}(t)}\right)
\end{aligned}
$$

where $\gamma_{i}^{\text {Req }}(t)$ is the SINR required for the hub $H_{i}$ to obtain the required data rate $R_{i}^{\text {Req }}$ considering the channel conditions and interference from neighboring WBASNs at time $t$. From Equation (12), we can determine $p_{i}^{\text {Req }}(t)$ as follows:

$$
p_{i}^{\text {Req }}(t)=\max \left[p_{\text {min }}, \min \left[p_{\max },\left(2^{\frac{R_{i}^{\text {Req }}}{B}}-1\right) \times \frac{p_{i}(t)}{\gamma_{i}(t)}\right]\right]
$$

where $p_{\max }$ and $p_{\min }$ are the predetermined maximum and minimum transmission power, respectively.

6. $\quad H_{i}$ transmits information of $U_{i}(t)$ to neighboring WBASNs.

7. $H_{i}$ computes the next target data rate using Equation (14) (defined below), with the received $U_{i}(t)$ information and the Cucker-Smale model, as follows:

$$
U_{i}(t+1)-U_{i}(t)=\frac{\lambda}{N} \sum_{j=1}^{N} \psi\left(\left|x_{j}-x_{i}\right|\right) \cdot\left(U_{j}(t)-U_{i}(t)\right)
$$

If any WBASN is out of communication range, $\psi(|\cdot|)=0$. Assuming $\psi\left(\left|x_{j}-x_{i}\right|\right)=1_{\left|x_{j}-x_{i}\right| \leq r}$ and $\lambda=1$, Equation (14) can be expressed simply, as the following equation:

$$
U_{i}(t+1)=\frac{1}{\left|N_{i}\right|} \sum_{j \in N_{i}} U_{j}(t)
$$

where $N_{i}$ is a set of WBASNs neighboring $H_{i}$, and $|\cdot|$ denotes the cardinality of the corresponding set.

8. If the difference between $U_{i}(t+1)$ and $U_{i}(t)$ is very small, i.e., $\left|U_{i}(t+1)-U_{i}(t)\right|<\epsilon$, where $\epsilon$ is a very small predefined constant, $H_{i}$ determines that the utility has converged and does not adjust the transmission power. 
9. $H_{i}$ determines the transmission power to be used at the next time interval $p_{i}(t+1)$ based on the calculated target utility $U_{i}(t+1) \cdot p_{i}(t+1)$ can be expressed as a single Equation (16) based on Equations (1), (2), and (11), with the same method used to define Equation (13).

$$
p_{i}(t+1)=\max \left\{p_{\text {min }}, \min \left\{p_{\text {max }}, \frac{p_{i}(t)}{\gamma_{i}(t)} \times\left(2^{\frac{R_{i}^{R e q} \ln \left(-\frac{1}{c} \ln \left(\alpha+e^{-c}-\alpha U_{i}(t+1)\right)\right)^{-b}+R_{i}^{R e q}}{B}}-1\right)\right\}\right\}
$$

where $\alpha=e^{-c e^{-b}}-e^{-c}$.

Algorithm 1 is a pseudo-code of the FTPC-U algorithm. During the execution of the proposed algorithm, it is possible to prevent sensor nodes from wasting transmission power in each WBASN, using Equation (13). It is also possible to synchronize the utilities of the WBASNs dispersively, using Equation (16). The data rate of the coordinator $H_{i}$ which is dependent on the user $i$ is related to the transmission power of all other neighboring sensor nodes $S N_{j}\left(\forall j \in N_{i}, j \neq i\right)$. Accordingly, adjusting the transmission power of one sensor node affects the data rate of all other coordinators. Therefore, the operating procedure of the proposed algorithm must be repeated, such that the utility value converges completely. Figure 6 is a schematic diagram of the operational procedure detailed above. Table 1 shows a comparison between elements used in the Cucker-Smale model and the proposed algorithm.

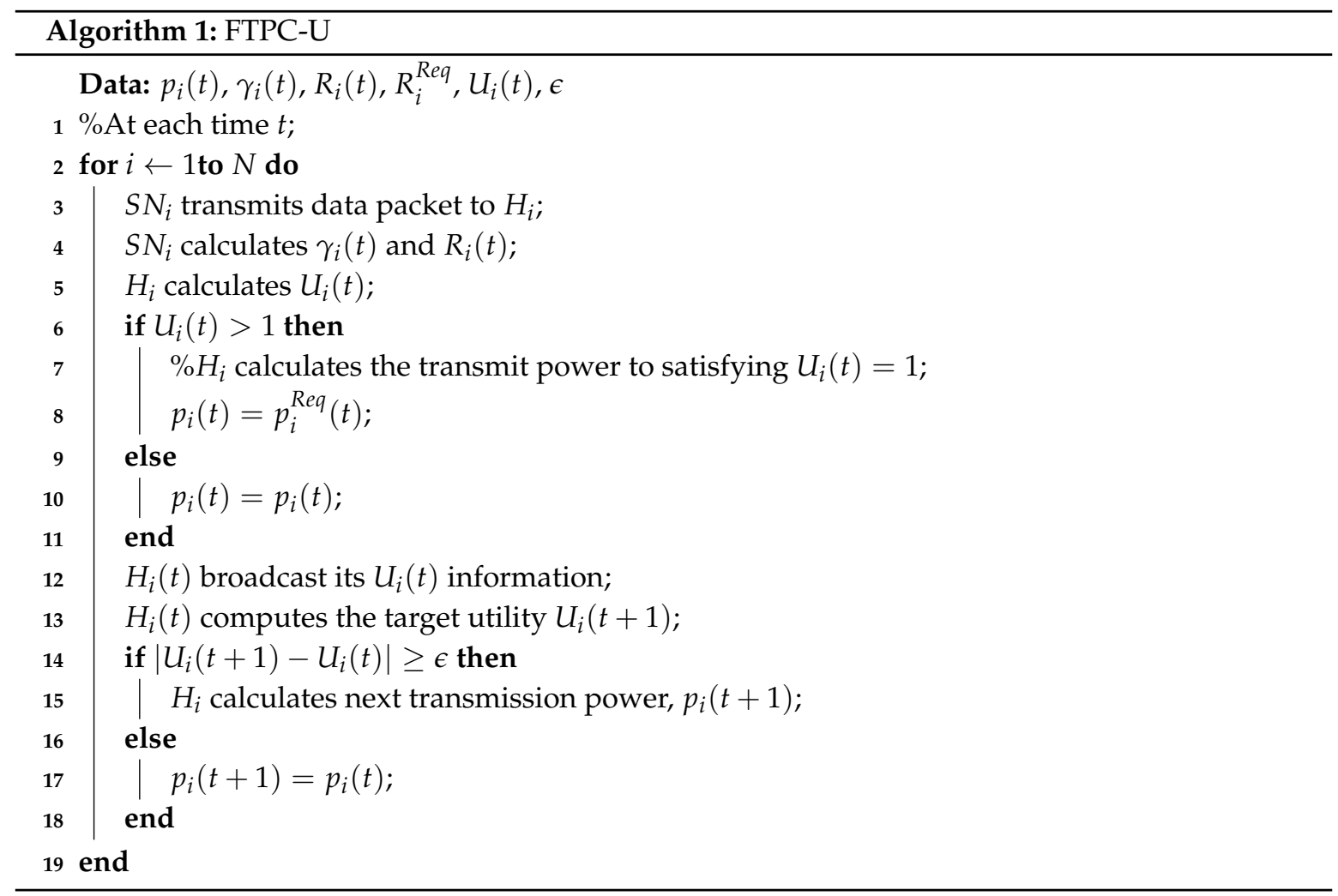


Table 1. Comparison between elements used in the Cucker-Smale model and the proposed algorithm. FTPC-U, locking-based transmission power control with utility.

\begin{tabular}{|c|c|c|}
\hline Elements & Cucker-Smale Model & FTPC-U \\
\hline Adjusted parameter & Velocity & Transmission power \\
\hline Communication range function & $\psi(|\cdot|)$ & $1_{\left|x_{j}-x_{i}\right|<r}$ \\
\hline Comparison of algorithm model & $\frac{d v_{i}(t)}{d t}=\frac{\lambda}{N} \sum_{j=1}^{N} \psi\left(\left|x_{j}-x_{i}\right|\right)\left(v_{j}(t)-v_{i}(t)\right)$ & $\frac{d U_{i}(t)}{d t}=\frac{1}{N_{i}} \sum_{j \in N_{i}} U_{j}(t)$ \\
\hline Objective to converge & Velocity & Utility \\
\hline
\end{tabular}

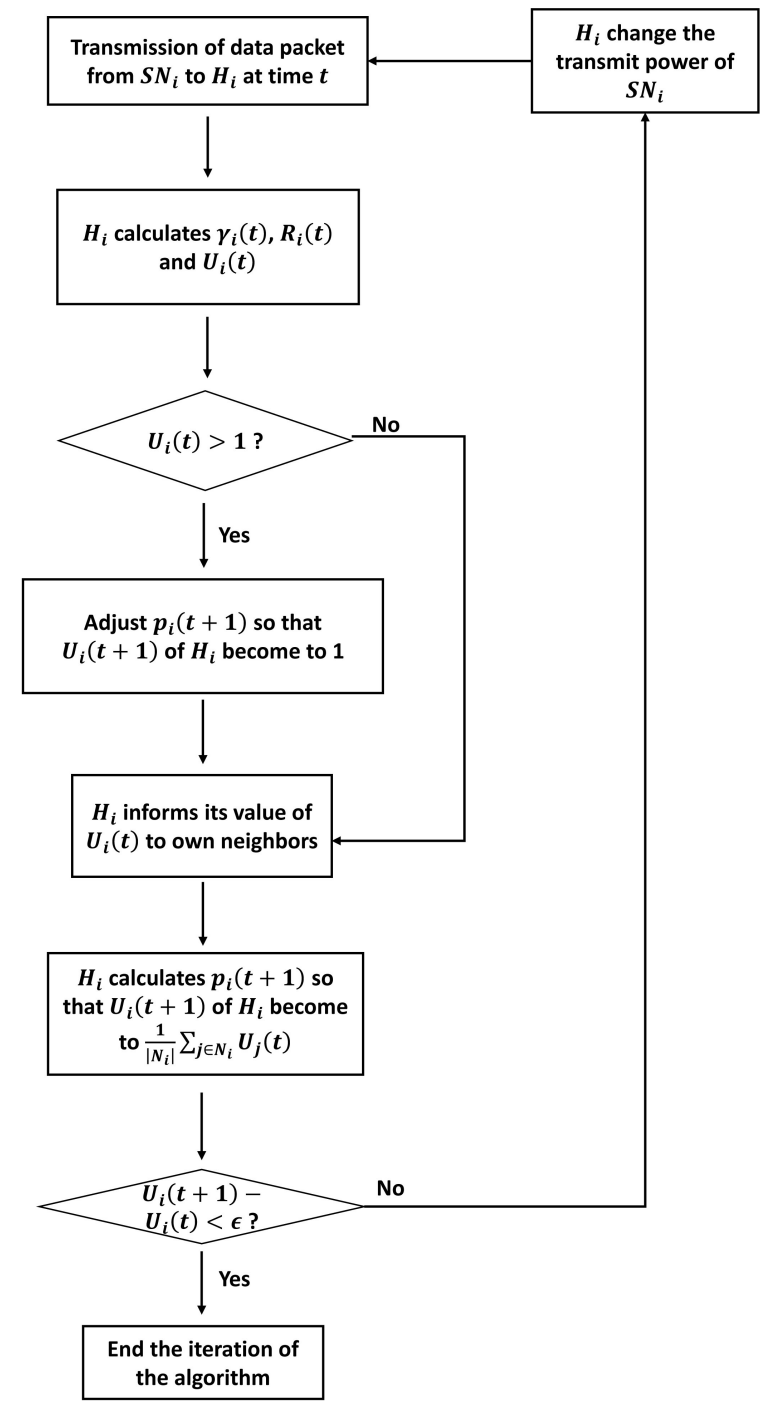

Figure 6. Operational procedure of the proposed algorithm.

\subsection{Convergence of the FTPC-U Algorithm}

In this section, to show the stability of the proposed algorithm, we prove its convergence. First, we consider Equation (14) in the continuous time domain, using the following equation:

$$
U_{i}(t+1)-U_{i}(t)=\frac{d U_{i}(t)}{d t}=\frac{\lambda}{N} \sum_{j=1}^{N} \psi\left(\left|x_{j}-x_{i}\right|\right) \cdot\left(U_{j}(t)-U_{i}(t)\right)
$$


The center of $U_{i}(t), U_{c}(t)$, is given by:

$$
U_{c}(t):=\frac{1}{N} \sum_{i=1}^{N} U_{i}(t)
$$

By differentiating Equation (18), we obtain the following:

$$
\begin{aligned}
\frac{d U_{c}(t)}{d t} & =\frac{1}{N} \sum_{i=1}^{N} \frac{d U_{i}(t)}{d t} \\
& =\frac{1}{N} \sum_{i=1}^{N} \sum_{j=1}^{N} \frac{\lambda}{N} \psi\left(\left|x_{j}-x_{i}\right|\right) \cdot\left(U_{j}(t)-U_{i}(t)\right) \\
& =\frac{\lambda}{N^{2}} \sum_{i=1}^{N} \sum_{j=1}^{N} \psi\left(\left|x_{j}-x_{i}\right|\right) \cdot\left(U_{j}(t)-U_{i}(t)\right) \\
& =\frac{\lambda}{2 N^{2}} \sum_{i=1}^{N} \sum_{j=1}^{N} \psi\left(\left|x_{j}-x_{i}\right|\right) \cdot\left(U_{j}(t)-U_{j}(t)+U_{i}(t)-U_{i}(t)\right) \\
& =0
\end{aligned}
$$

The derivative value in Equation (19) becomes zero, indicating that $U_{c}(t)=U_{c}(0) . \hat{U}_{i}(t)$, the fluctuations around $U_{\mathcal{c}}(0)$, can be defined as follows:

$$
\hat{U}_{i}(t):=U_{i}(t)-U_{c}(0)
$$

From Equations (18) and (20), we have:

$$
\sum_{i=1}^{N} \hat{U}_{i}(t)=0
$$

Here, we define $\mathbf{U}(t)$ as $\mathbf{U}(t):=\left\{\hat{U}_{1}(t), \hat{U}_{2}(t), \cdots, \hat{U}_{N}(t)\right\} \in \mathbb{R}^{N}$, which is the set of $\hat{U}_{i}(t)$. The $L^{2}$-norm of this set, which denotes quantities proportional to the standard deviations of $\mathbf{U}(t)$, is given by:

$$
\|\mathbf{U}(t)\|=\left(\sum_{i=1}^{N}\left|\hat{U}_{i}(t)\right|^{2}\right)^{\frac{1}{2}}
$$

Substituting Equation (20) into Equation (17) yields:

$$
\begin{aligned}
\frac{d\left|\hat{U}_{i}(t)\right|^{2}}{d t} & =2 \hat{U}_{i}(t) \cdot \frac{d\left|\hat{U}_{i}(t)\right|}{d t} \\
& =2 \hat{U}_{i}(t) \cdot \frac{\lambda}{N} \sum_{j=1}^{N} \psi\left(\left|x_{j}-x_{i}\right|\right) \cdot\left(\hat{U}_{j}(t)-\hat{U}_{i}(t)\right)
\end{aligned}
$$

We calculate the derivative of $\|\mathbf{U}(t)\|^{2}$ as follows:

$$
\begin{aligned}
\frac{d\|\mathbf{U}(t)\|^{2}}{d t} & =\frac{d}{d t} \sum_{i=1}^{N}\left|\hat{U}_{i}(t)\right|^{2}=\sum_{i=1}^{N} \frac{d\left|\hat{U}_{i}(t)\right|^{2}}{d t} \\
& =\frac{\lambda}{N} \sum_{i=1}^{N} 2 \hat{U}_{i}(t) \sum_{j=1}^{N} \psi\left(\left|x_{j}-x_{i}\right|\right) \cdot\left(\hat{U}_{j}(t)-\hat{U}_{i}(t)\right) \\
& =-\frac{\lambda}{N} \sum_{i=1}^{N} \sum_{j=1}^{N} \psi\left(\left|x_{j}-x_{i}\right|\right) \cdot\left(2 \hat{U}_{j}(t)^{2}-2 \hat{U}_{j}(t) \hat{U}_{i}(t)\right) \\
& =-\frac{\lambda}{N} \sum_{i=1}^{N} \sum_{j=1}^{N} \psi\left(\left|x_{j}-x_{i}\right|\right) \cdot\left|\hat{U}_{j}(t)-\hat{U}_{i}(t)\right|^{2}
\end{aligned}
$$


We assume that the maximum difference in location between any two WBASNs in the network is given by $x_{\max }=\max _{i, j}\left|x_{j}-x_{i}\right|$. Because $\psi(\cdot)$ is a non-negative and non-increasing function, we can rewrite Equation (24) as,

$$
\begin{aligned}
\frac{d\|\mathbf{U}(t)\|^{2}}{d t} & \leq-\frac{\lambda}{N} \sum_{i=1}^{N} \sum_{j=1}^{N} \psi\left(x_{\max }\right) \cdot\left|\hat{U}_{j}(t)-\hat{U}_{i}(t)\right|^{2} \\
& =-\frac{\lambda}{N} \psi\left(x_{\max }\right) \sum_{i=1}^{N} \sum_{j=1}^{N}\left(\hat{U}_{j}(t)^{2}+\hat{U}_{i}(t)^{2}-2 \hat{U}_{j}(t) \hat{U}_{i}(t)\right) \\
& =-\frac{\lambda}{N} \psi\left(x_{\max }\right)\left(\sum_{i=1}^{N} \sum_{j=1}^{N} 2 \hat{U}_{i}(t)^{2}-2 \sum_{i=1}^{N} \hat{U}_{i}(t) \sum_{j=1}^{N} \hat{U}_{j}(t)\right) \\
& =-\frac{\lambda}{N} \psi\left(x_{\max }\right)\left(2 N\|\mathbf{U}(t)\|^{2}-0\right) \\
& =-2 \lambda \psi\left(x_{\max }\right)\|\mathbf{U}(t)\|^{2}
\end{aligned}
$$

By solving Equation (25), we can get the following inequalities:

$$
\begin{gathered}
\|\mathbf{U}(t)\|^{2} \leq\|\mathbf{U}(0)\|^{2} e^{-2 \lambda \psi\left(x_{\max }\right) t} \\
\|\mathbf{U}(t)\| \leq\|\mathbf{U}(0)\| e^{-\lambda \psi\left(x_{\max }\right) t}
\end{gathered}
$$

which dictate that the proposed algorithm achieves exponential convergence.

\section{Simulation Results}

In this section, we evaluate the performance of the proposed algorithm using Monte-Carlo simulations. We assume the network topology proposed in Section 2. Each WBASN operates in beacon mode according to the IEEE 802.15.6 standard, and exchanges utility information. A hospital waiting room was considered, as a dense environment where interference could occur. Figure 7 depicts the network environment, assuming a hospital waiting room. The area is set to $14 \mathrm{~m} \times 4 \mathrm{~m}$, where the maximum number of WBASNs is 48 . In all subsequent simulations, we will use a logarithmically-shaped utility function, with control parameters $b=1$ and $c=9$. Table 2 summarizes the applications used in the simulations detailed in this paper, and considers the requirements for various services used in the WBASN [1,46,47].

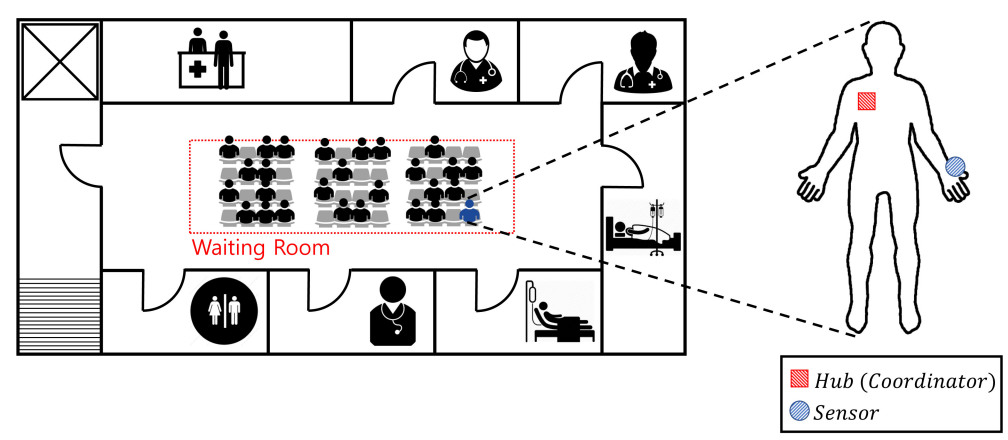

Figure 7. Network environment, assuming a hospital waiting room. 
Table 2. Type of application used in the WBASN and required data rate.

\begin{tabular}{cc}
\hline Application Name & Required Data Rate \\
\hline ECG & $288 \mathrm{kbps}$ \\
Artificial retina & $700 \mathrm{kbps}$ \\
Capsule endoscope & $1 \mathrm{Mbps}$ \\
EMG & $1.536 \mathrm{Mbps}$ \\
\hline
\end{tabular}

The simulation considers an uplink situation where measured data is transmitted from the sensor node to the coordinator. The channel gain in decibels is given by $g_{i j}=16.7 \log _{10} d-0.45$, where $d$ is the distance [48]. Table 3 summarizes the commonly-used parameters in this paper.

Table 3. Commonly-used simulation parameters.

\begin{tabular}{cc}
\hline Parameter & Value \\
\hline Network size & $14 \mathrm{~m} \times 14 \mathrm{~m}$ \\
Size of each seat & $1 \mathrm{~m} \times 1 \mathrm{~m}$ \\
Location of hub & Center of assigned seat \\
Location of sensor node & Uniformly random distribution within the area of each seat \\
Initial transmission power & Uniformly random allocation in $(-14,-6), \mathrm{dBm}$ \\
Bandwidth & $1 \mathrm{MHz}$ \\
Path loss model & $g_{i j}=16.7 \log _{10} d-0.45($ in $\mathrm{dB}, d=$ distance) \\
Convergence error $(\epsilon)$ & $10^{-4}$ \\
\hline
\end{tabular}

Furthermore, we compared the performance of the proposed algorithm with two algorithms: PAPU and transmission power control for body area network (TPC-BAN). PAPU controls transmission power based on game theory and aims to increase the transmission rate within a given power limit, and calculates the Nash equilibrium using the best response concept to determine the optimized transmission power value by distributed processing [7]. This algorithm is adapted to update the transmit power according to the occurrence of the event. There are two kinds of events. The first one occurs when the transmission power is adjusted in the other network, and the second event occurs when the SINR variation according to the time is equal to or greater than a predetermined threshold value. When an event occurs, the hub changes the transmit power according to the best response and informs the neighboring network of the change of the transmit power of the hub by broadcasting. TPC-BAN controls transmission power using the detected RSSI [23]. In TPC-BAN, the hub updates its own average RSSI by giving weighted parameter in order to mitigate the effects of instantaneous channel mutation. The hub determines whether to control transmission power or not by comparing the average RSSI value with the predetermined target reception power. If the average RSSI value is located in the offset range of the target RSSI, the hub does not change the power of sensor node. However, if the average RSSI value is out of the offset range of the target RSSI, the hub changes the transmission power of the sensor node. The algorithm periodically updates its average RSSI and adjusts the transmit power of the sensor nodes until it reaches the offset range of the target RSSI.

To verify the performance of the proposed algorithm, we create a network environment where nine WBASNs are placed close together, at specific locations, as shown in Figure 8. Table 4 shows information of applications used by each WBASN in this environment.

Figure 9 shows the changes in performance for each user over time, using the proposed algorithm in the network environment shown in Figure 8 and Table 4. As shown in Figure 9a, WBASN $W B A S N_{2}$, and $W B A S N_{3}$ have a low required data rate $(288 \mathrm{kbps})$. Thus, each coordinator reduces the transmission power of the corresponding sensor nodes, which do not require a large transmission power. In the case of $W B A S N_{9}$, the coordinator increases the transmission power of the sensor node, which has a high required data rate $(1.536 \mathrm{Mbps})$. By adjusting the transmission power, the data rate of each WBASN converges to a specific value, based on the required data rate, as shown in Figure 9b. 
As shown in Figure 9c, the utility of each user converges to the same value. As shown in Figure 9d, when the proposed algorithm is applied, only the transmission power needed for data transfer at the required data rate is consumed. Hence, it shows that transmission using the proposed algorithm has a high energy-efficiency.
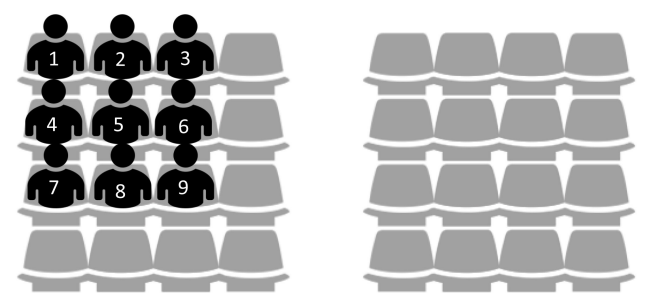

Figure 8. Location distribution of each WBASN in the performance-evaluation-over-time.

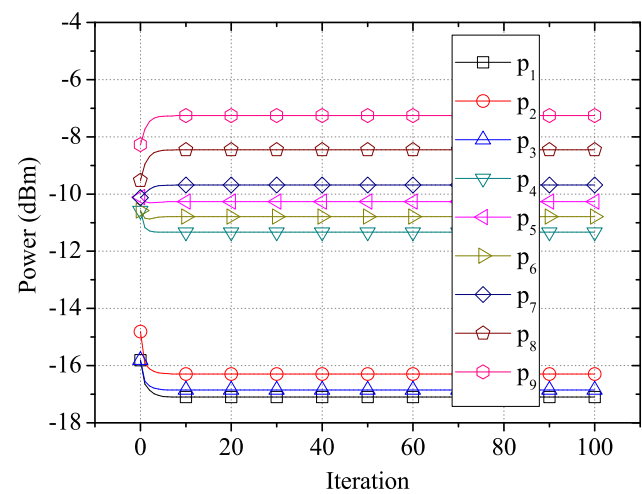

(a)

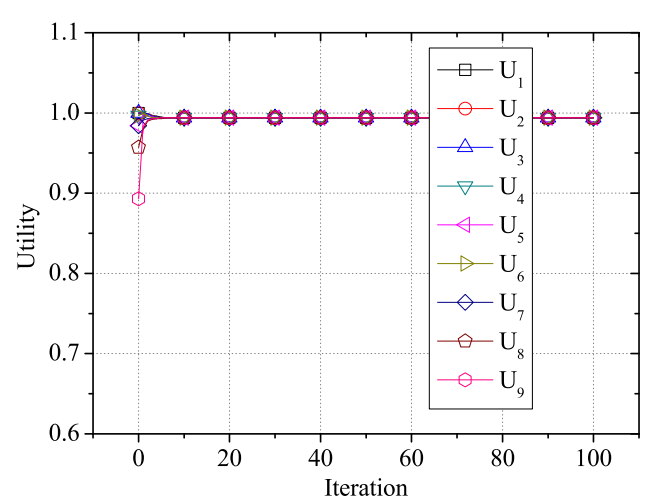

(c)

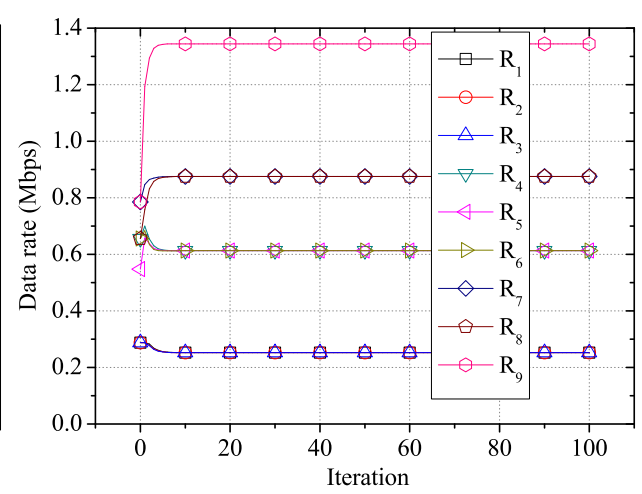

(b)

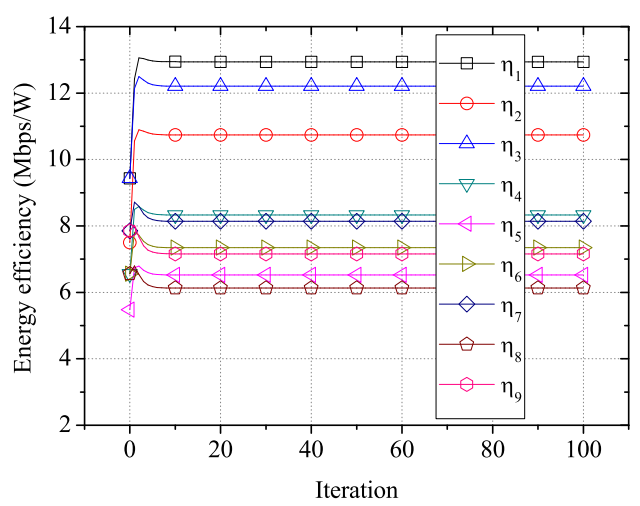

(d)

Figure 9. Performance of FTPC-U over time. (a) Transmission power over time; (b) data rate over time; (c) utility over time; (d) energy efficiency over time.

Table 4. Applications used by WBASNs in the performance-evaluation-over-time.

\begin{tabular}{cccc}
\hline WBASN ID & Application & WBASN ID & Application \\
\hline 1 & ECG (288 kbps) & 6 & Artificial retina \\
2 & ECG & 7 & Capsule endoscope (1 Mbps) \\
3 & ECG & 8 & Capsule endoscope \\
4 & Artificial retina (700 kbps) & 9 & EMG (1.536 Mbps) \\
5 & Artificial retina & - & - \\
\hline
\end{tabular}


Figure 10 compares the performance of the proposed algorithm with PAPU and TPC-BAN. With the distribution of locations and applications as shown in Figure 8 and Table 4, we conduct simulations to obtain an average performance value of each WBASN over time when the proposed algorithm, PAPU, and TPC-BAN are employed. The purposes of PAPU and TPC-BAN are to increase the overall data rate of the entire node and to satisfy global target RSSI value, respectively, while the proposed scheme aims to increase the fairness of the QoS, and decrease the transmission power of each user, simultaneously. Therefore, when using the proposed scheme, lower transmission powers and average data rates are observed as shown in Figure 10a,b, and a higher utility value is observed as shown in Figure 10c. Since with the proposed algorithm each user has a high average utility, the number of users with a satisfactory QoS is greater than when PAPU or TPC-BAN is used, as shown in Figure 10d. In addition, since the proposed scheme requires a lower transmission power on average, energy efficiency is high, as shown in Figure 10e.

To evaluate the QoS fairness of network users, we use Jain's fairness index [49], defined as follows:

$$
\mathcal{J}\left(x_{1}, x_{2}, \cdots, x_{N}\right)=\frac{\left(\sum_{i=1}^{N} x_{i}\right)^{2}}{N \sum_{i=1}^{N} x_{i}^{2}}
$$

where $x_{i}=R_{i}(t) / R_{i}^{R e q}$. Jain's fairness index defines a variable $x_{i}$ that indicates how close the current data rate $R_{i}(t)$ is to the required data rate $R_{i}^{R e q}$ for each of the $N$ users in the network. As the difference in $x_{i}$ is reduced, the QoS for each user is satisfied fairly, and the value of Jain's fairness index approaches one. Figure 10f is a graph comparing the Jain's fairness index of the proposed method with the index obtained using PAPU and TPC-BAN. Since the proposed algorithm synchronizes the utility, higher QoS fairness is observed than with PAPU and TPC-BAN.

Figure 11 shows the average performance of FTPC-U, PAPU, and TPC-BAN according to the number of nodes. We change the number of nodes from 2-24, placing each node in a $14 \mathrm{~m} \times 4 \mathrm{~m}$ space, as shown in Figure 7. Each node is randomly assigned one of the applications listed in Table 2. Figure 11a shows the transmission power according to the number of nodes. In this case, the overall interference increases as the number of nodes increases. With PAPU and TPC-BAN, the hub tries to reduce the transmission power of the sensor node as the interference increases. However, with FTPC-U, the hub adjusts the transmission power of each sensor node using Equation (16), for synchronization of the utilities. In this case, if a decrease in SINR caused by the increase in interference from Equation (16) occurs, the transmission power of the sensor node is increased. Figure $11 \mathrm{~b}$ is a graph showing the data rate according to the number of nodes. When the number of nodes is small, we observe a high data rate, which decreases as the number of nodes increases, using PAPU or TPC-BAN. In contrast, with FTPC-U, we observe a low data rate when the number of nodes is small, because the required data rate can be achieved without a high transmission power. We note that Figure 11c shows an average utility of one, until the number of nodes is about 12 . This shows that the actual data rate is close to the required data rate. Therefore, even though the data rate observed in Figure $11 \mathrm{~b}$ is low, the user does not perceive a deterioration in performance. We note from Figure 11c that the effect of the number of nodes on performance is less prevalent using FTPC-U, which controls the transmission power considering the interference caused by the increase in the number of nodes, and the data rate of each user, than it is with PAPU or TPC-BAN. Therefore, as shown in Figure 11d, the number of users with a satisfactory QoS is kept high with FTPC-U, but decreases gradually with PAPU and TPC-BAN. Since the minimum power required for a satisfactory QoS is used with the proposed algorithm, the energy efficiency is high as shown in Figure 11e. Figure 11f shows the QoS fairness according to the number of nodes. FTPC-U shows higher QoS fairness than PAPU and TPC-BAN because it adapts the synchronization of utilities. 


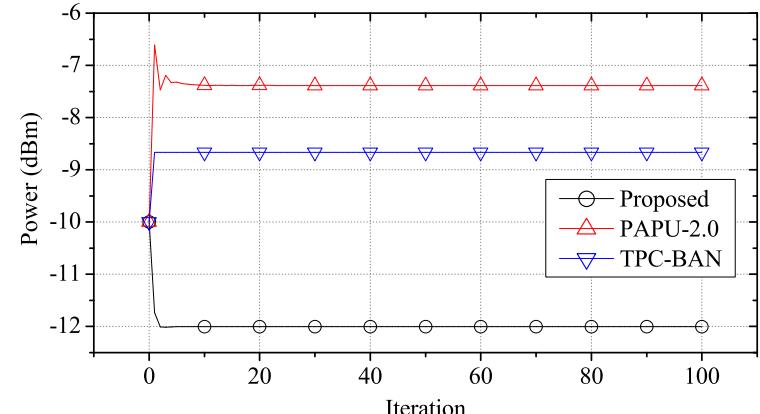

(a)

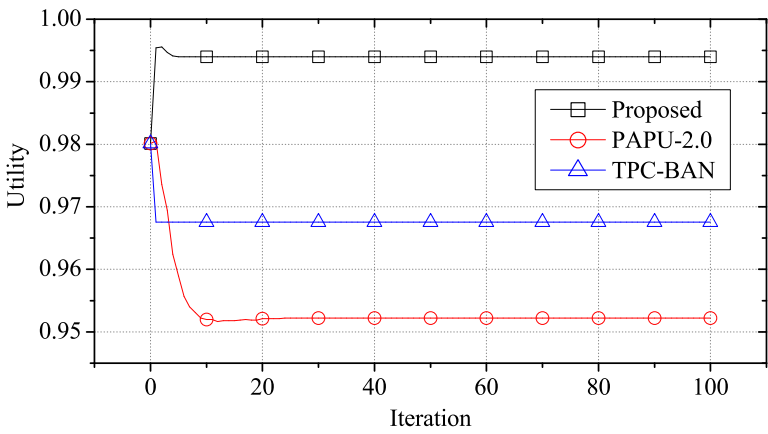

(c)

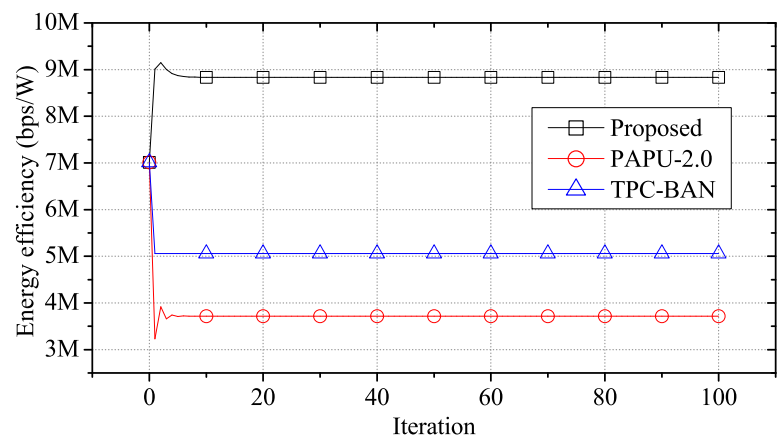

(e)

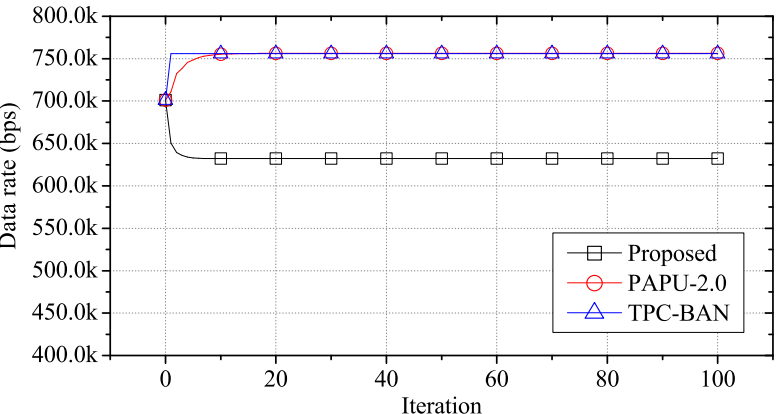

(b)

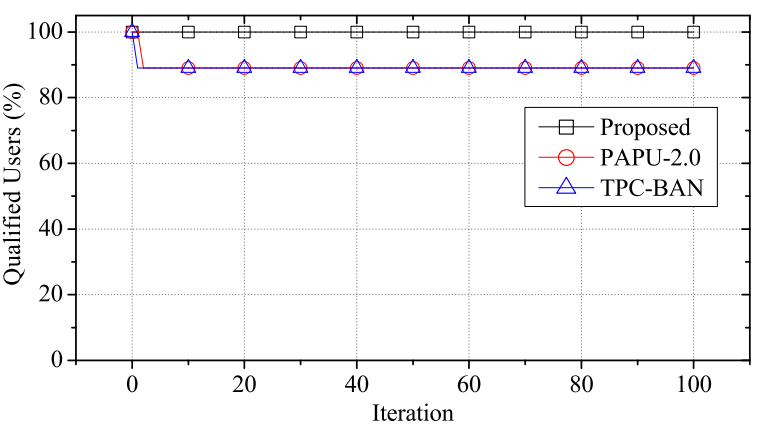

(d)

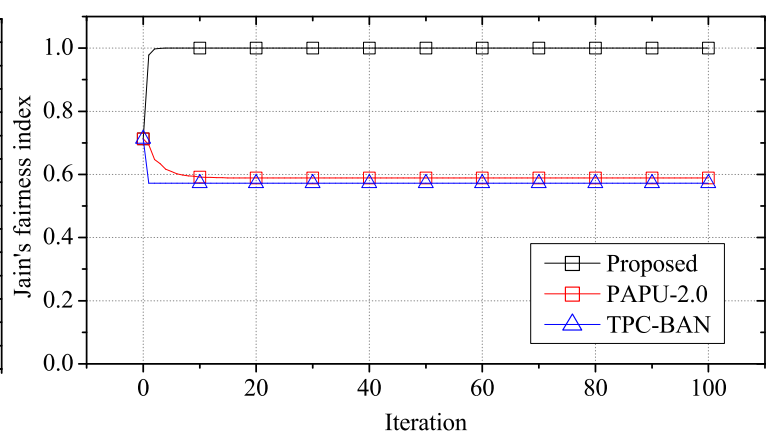

(f)

Figure 10. Performance of FTPC-U compared with PAPU and TPC-BAN over time. (a) Change in average transmit power over time; (b) change in average data rate over time; (c) change in average utility over time; (d) change in qualified users over time; (e) change in average energy efficiency over time; (f) change in fairness over time. PAPU, proactive power update; TPC-BAN, transmission power control for body area network.

Figure 12 shows the order that each WBASN arrives to the network environment, and its corresponding location information. In the initial stage, we assume that only four WBASNs, $W B A S N_{1}-W B A S N_{4}$, exist. Then, one WBASN sequentially enters when the number of iterations increases by a 100 , so that up to nine WBASNs are in the network environment. To evaluate performance in dense situations, an arriving WBASN is placed in a location adjacent to an existing WBASN. Table 5 shows the application information of each WBASN in this environment.

Figure 13 shows the change in performance according to the entry of WBASNs, when the network environment detailed in Figure 12 and Table 5 is applied. Figure 13a,b shows the changes in transmission power and data rate when a new WBASN enters an existing network environment. Here, when the new WBASN enters, a temporary change in interference may cause a temporary change to the transmission power, and a decrease in the data rate. In addition, the utility of each WBASN can be reduced temporarily, as shown in Figure 13c. Due to the instantaneous change in 
utility, the number of users with a satisfactory QoS temporarily decreases, as shown in Figure 13d. As shown in Figure 13e, high energy efficiency is observed using the proposed algorithm, because only as much transmission power as required is consumed. Figure $13 \mathrm{f}$ shows the QoS fairness in the entry environment, indicating that the proposed scheme achieves high QoS fairness through utility synchronization.

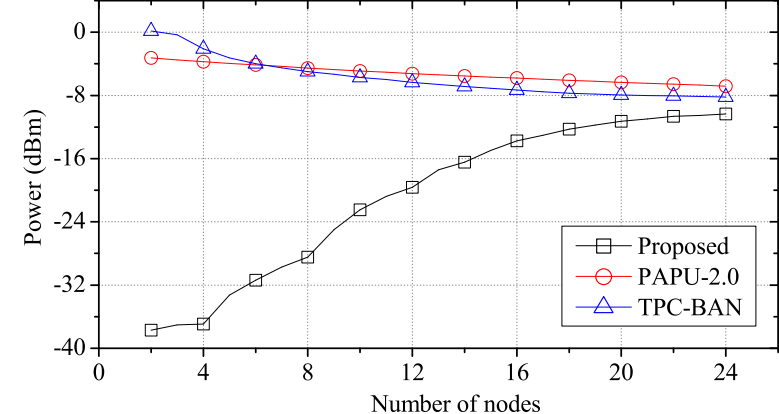

(a)

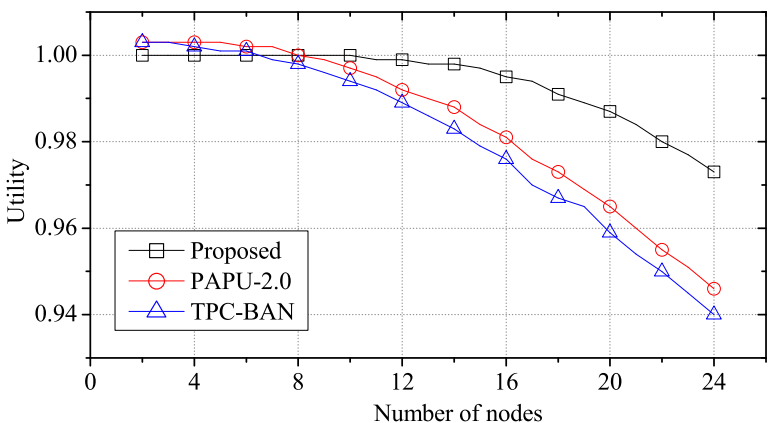

(c)

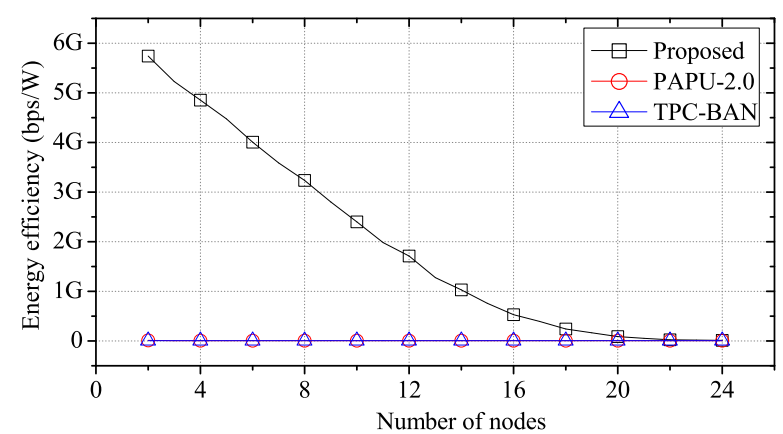

(e)

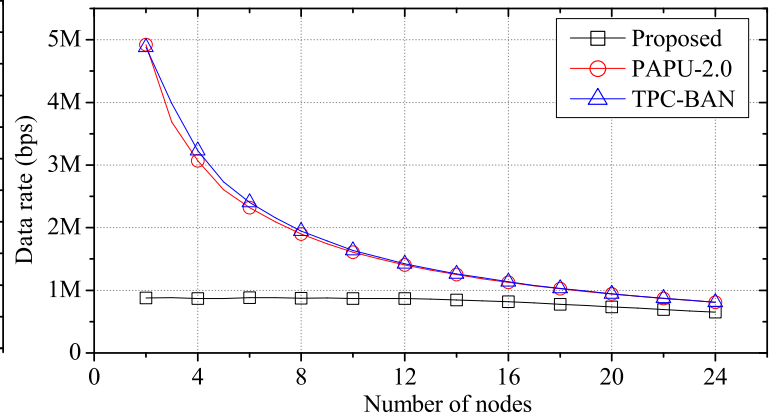

(b)

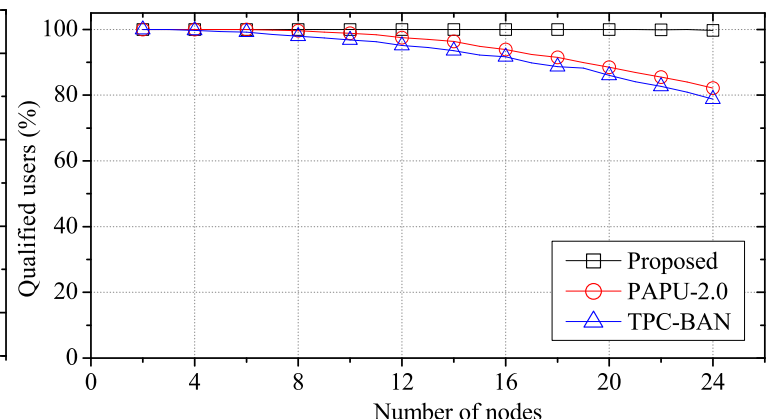

(d)

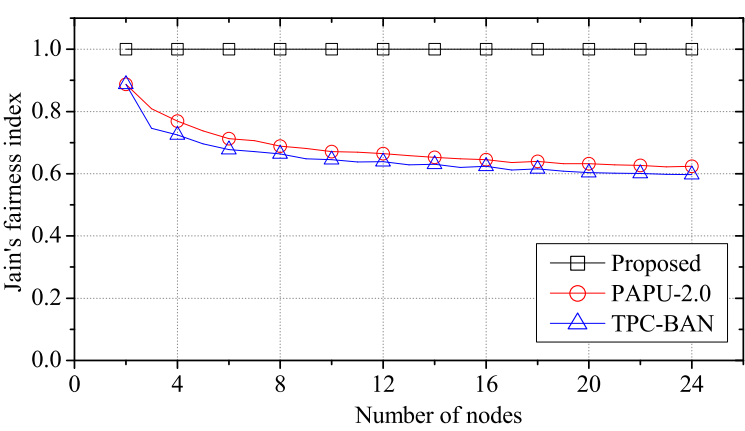

(f)

Figure 11. Performance of FTPC-U compared with PAPU and TPC-BAN according to the number of nodes. (a) Change in average transmit power; (b) change in average data rate; (c) change in average utility; (d) change in qualified users; (e) change in average energy efficiency; (f) change in fairness. 

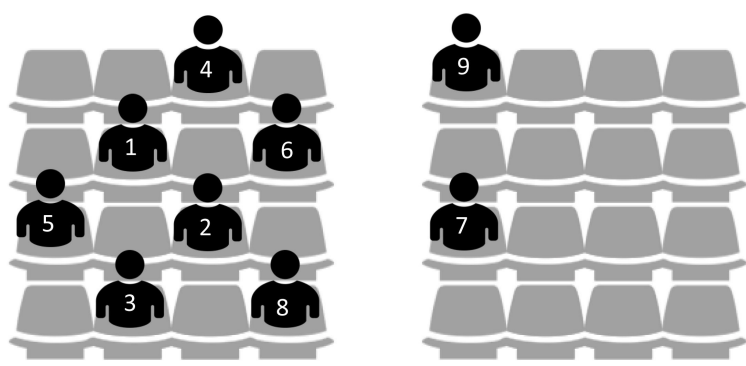

Figure 12. Location distribution of each WBASN in the performance-evaluation-with-entry model.

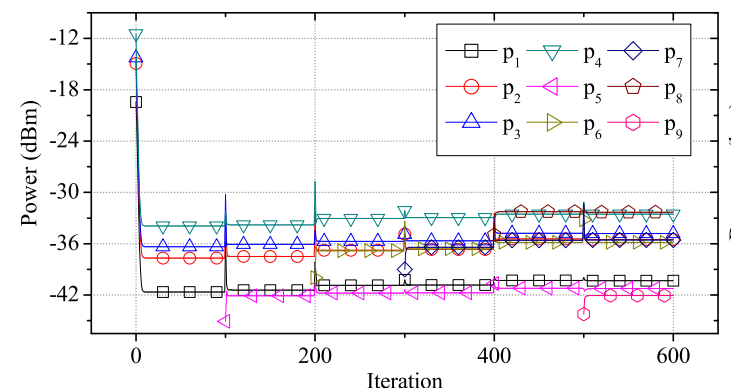

(a)

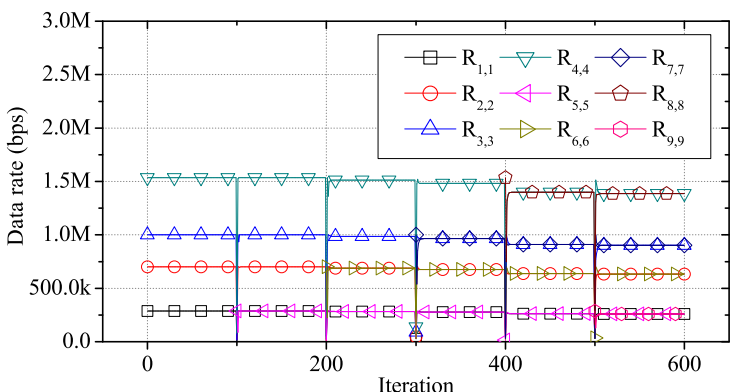

(b)

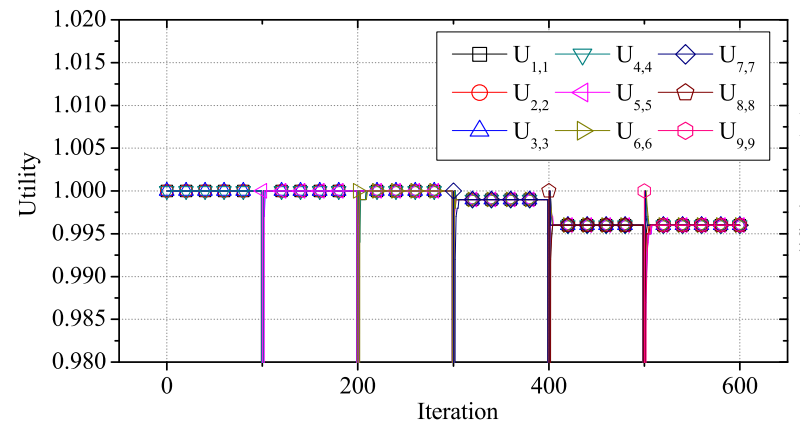

(c)

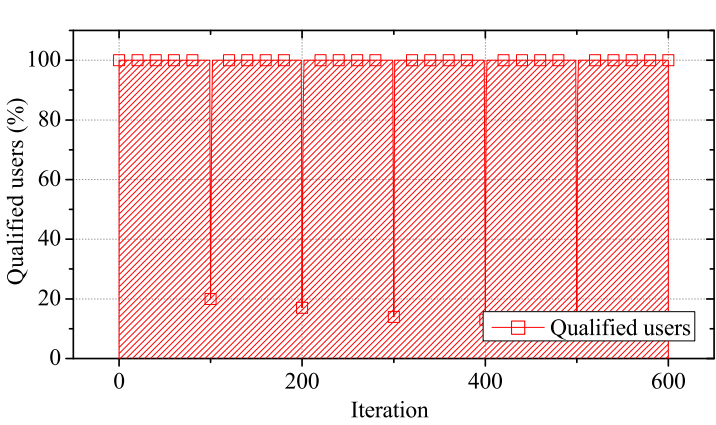

(d)

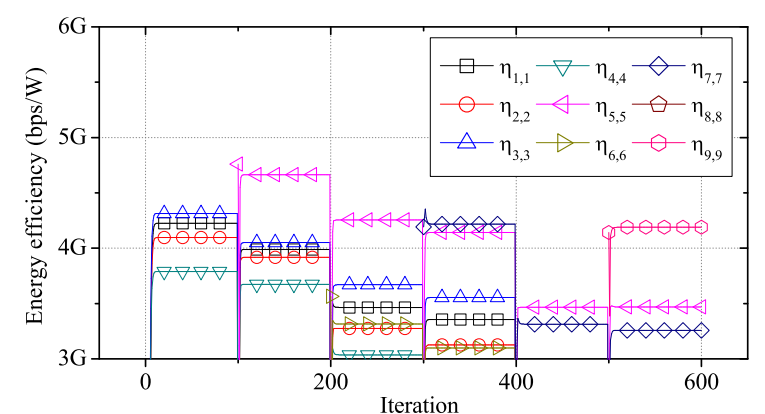

(e)

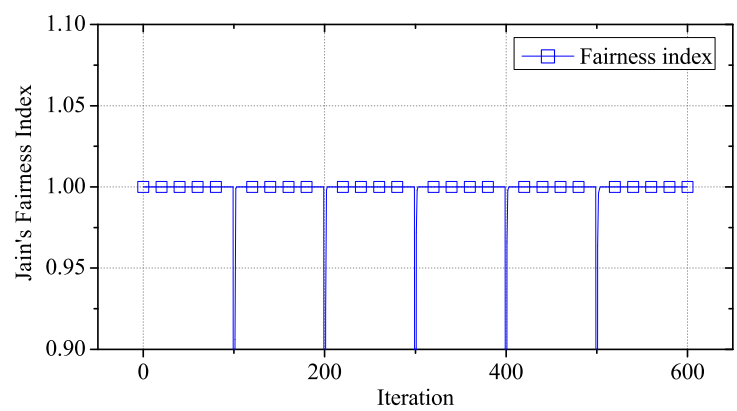

(f)

Figure 13. Performance of FTPC-U over time in the entry model. (a) Change in transmit power over time in the entry model; (b) change in data rate over time in the entry model; (c) change in utility over time in the entry model; (d) change in qualified users over time in the entry model; (e) change in energy efficiency over time in the entry model; (f) change in fairness over time in the entry model. 
Table 5. Applications used by WBASNs in the performance-evaluation-with-entry model.

\begin{tabular}{cccc}
\hline WBASN ID & Application & WBASN ID & Application \\
\hline 1 & ECG (288 kbps) & 6 & Artificial retina \\
2 & Artificial retina (700 kbps) & 7 & Capsule endoscope (1 Mbps) \\
3 & Capsule endoscope (1 Mbps) & 8 & EMG \\
4 & EMG (1.536 Mbps) & 9 & ECG \\
5 & ECG & - & - \\
\hline
\end{tabular}

Figure 14 shows the average performance of FTPC-U, PAPU, and TPC-BAN when a new WBASN enters an existing network environment. With the entry model and applications as shown in Figure 12 and Table 5, we evaluate the average performance value of each WBASN with FTPC-U, PAPU, and TPC-BAN. As shown in Figure 14a, transmission power is not consumed unnecessarily with FTPC-U, as the influence of interference and required data rate are considered, whereas PAPU and TPC-BAN controls the transmission power to maximize the data rate and to satisfy global target RSSI, respectively. Therefore, as shown in Figure 14c, although a lower data rate is observed using FTPC-U than with PAPU or TPC-BAN, the converged utility value is almost the same in both cases. Moreover, since all of algorithms maintain a utility close to one, the number of users with a satisfactory QoS is also maintained at about $100 \%$ as shown in Figure $14 \mathrm{~d}$. Figure $14 \mathrm{e}$ shows the energy efficiency in the WBASN entry environment. FTPC-U has a significantly higher energy efficiency than PAPU or TPC-BAN, because it operates at low power. Figure $14 \mathrm{f}$ shows the QoS fairness for users in the WBASN entry environment. When PAPU or TPC-BAN is used, the QoS fairness of the users is reduced each time a new WBASN enters the network environment. However, if FTPC-U is used, Jain's fairness index is maintained at about one. In the entry environment, the performance temporarily decreases, because interference increases at the moment of entry. The overall performance degradation at this moment is larger with FTPC-U than with PAPU or TPC-BAN. This is because, with PAPU or TPC-BAN, the transmission power of most of the sensor nodes is adjusted to a relatively high level, however, with FTPC-U, many sensor nodes are adjusted to use a low transmission power, and sensor nodes with low transmission powers are more seriously affected by interference caused by a newly entering WBASN. Nevertheless, using FTPC-U, we observe high performance in the converged state. 


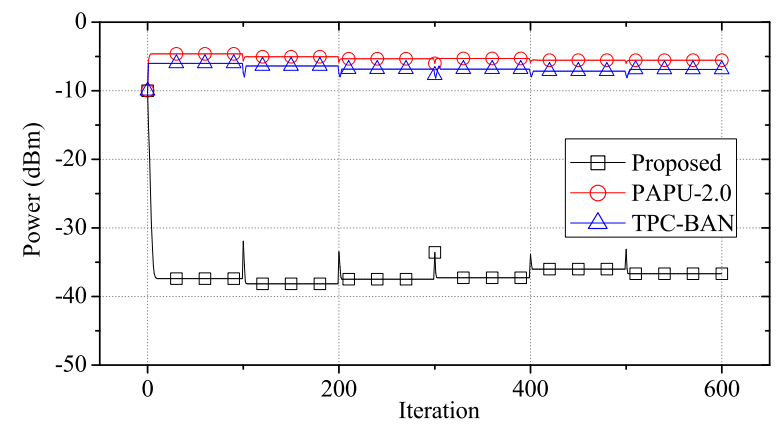

(a)

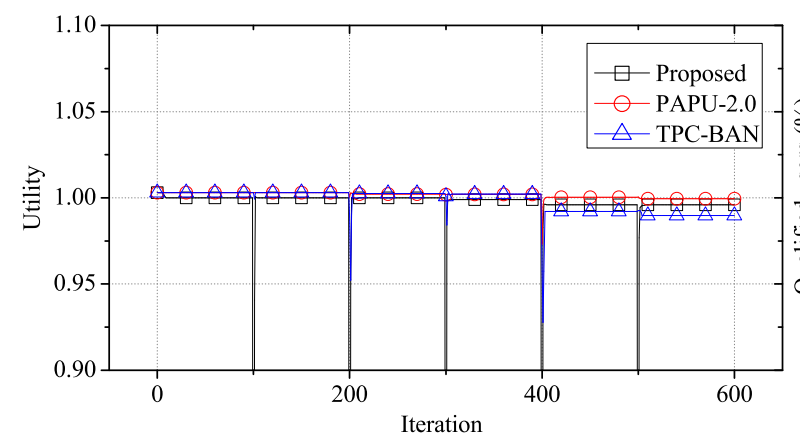

(c)

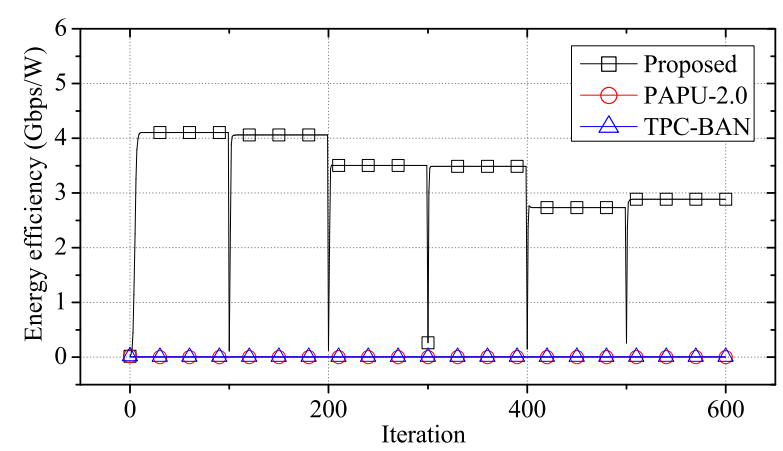

(e)

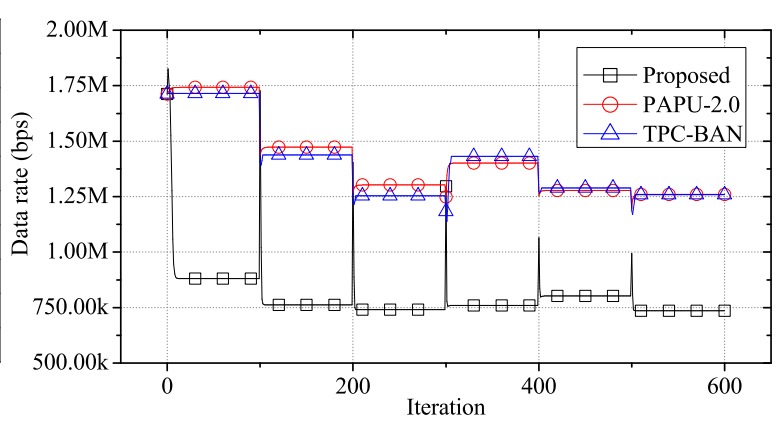

(b)

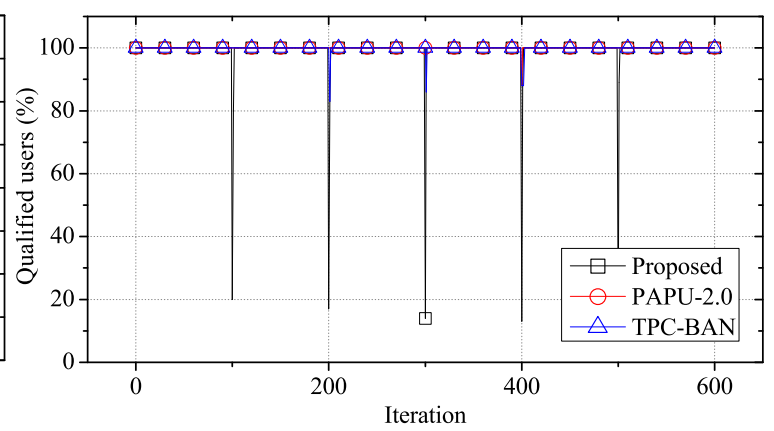

(d)

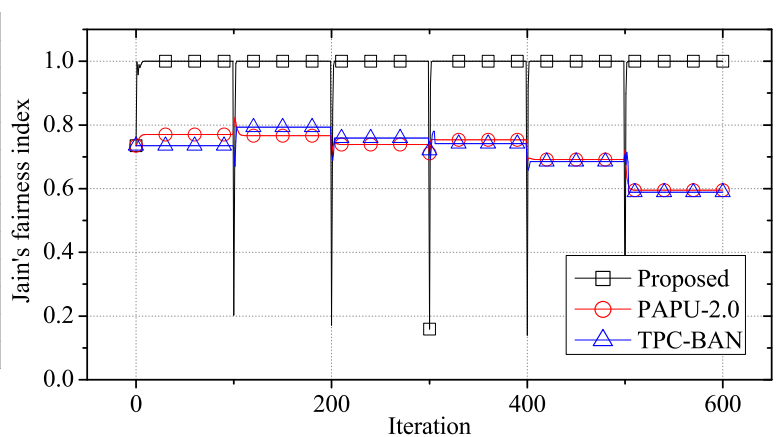

(f)

Figure 14. Performance of FTPC-U compared with PAPU and TPC-BAN over time in the entry environment. (a) Change in average transmit power; (b) change in average data rate; (c) change in average utility; (d) change in qualified users; (e) change in average energy efficiency; (f) change in fairness.

\section{Conclusions}

We introduced FTPC-U, which is a flocking-inspired algorithm for controlling transmission power using the Cucker-Smale model, to solve interference problems that occur in the WBASN environment and to guarantee QoS fairness between users. With the proposed algorithm, a fair QoS is guaranteed for each user, by calculating a utility that expresses the QoS satisfaction numerically and synchronizing this utility with those of neighboring WBASNs. Results of simulations show that FTPC-U operates stably in both static and dynamic states. Comparing to PAPU and TPC-BAN, FTPC-U consumes less power and exhibits higher or equal utility values. Improved energy efficiency and QoS fairness were observed with FTPC-U, in both static and entry environments, compared with PAPU and TPC-BAN. As a result, FTPC-U can effectively mitigate interference in dense environments and ensure that the QoS satisfaction value for each user is fair. In addition, we expect that using this algorithm, high performance can be achieved with other types of networks where various applications coexist in a dense and highly mobile environment. 
In this paper, we assumed that only one sensor node is associated with a WBASN. However, it is more natural to assume the coexistence of multiple sensor nodes associated with a WBASN. Each sensor node associated with a WBASN may have a different QoS requirement, and this leads to the scheduling issue in a WBASN. For this reason, it is necessary to consider QoS-fairness of multiple sensor nodes in a WBASN. Therefore, a study on the joint transmission power control and resource allocation algorithm for QoS-fairness of all users considering both intra-WBASN and inter-WBASN collisions would be our further work. In addition, the proposed algorithm assumes that all hubs and sensor nodes operate normally without any malicious node. However, the performance of the network may deteriorate by malicious nodes, which may cause interference increase, data collision and obstruction of convergence. We have a plan to extend the proposed algorithm considering the existence of malicious nodes.

Acknowledgments: This work was supported by the Human Resources Development (No. 20174030201810) of the Korea Institute of Energy Technology Evaluation and Planning (KETEP) grant funded by the Korea government Ministry of Trade, Industry and Energy and the Basic Science Research Program through the National Research Foundation of Korea (NRF) funded by the Ministry of Education, Science and Technology (No.2015R1D1A1A01060207).

Author Contributions: Chan-Jae Lee contributed the paper by deriving initial results of simulation and by making the draft version of the paper. Ji-Young Jung contributed the paper by correcting some errata in the draft version of the paper, deriving results of simulation using TPC-BAN, and writing the response letters. Jung-Ryun Lee was responsible for the main idea, theoretical analysis, coordination and proof reading of the paper.

Conflicts of Interest: The authors declare no conflict of interest.

\section{References}

1. Movassaghi, S.; Abolhasan, M.; Lipman, J.; Smith, D.; Jamalipour, A. Wireless body area networks: A survey. IEEE Commun. Surv. Tutor. 2014, 16, 1658-1686, doi:10.1109/SURV.2013.121313.00064.

2. Negra, R.; Jemili, I.; Belghith, A. Wireless body area networks: Applications and technologies. Procedia Comput. Sci. 2016, 83, 1274-1281, doi:10.1016/j.procs.2016.04.266.

3. Miranda, J.; Cabral, J.; Wagner, S.R.; Pedersen, C.F.; Ravelo, B.; Memon, M.; Mathiesen, M. An open platform for seamless sensor support in healthcare for the internet of things. Sensors 2016, 16, 2089, doi:10.3390/s16122089.

4. Arbia, D.B.; Alam, M.M.; Moullec, Y.L.; Hamida, E.B. Communication Challenges in on-Body and Body-to-Body Wearable Wireless Networks-A Connectivity Perspective. Technologies 2017, 5, 43, doi:10.3390/technologies5030043.

5. IEEE Computer Society. IEEE Standard for Local and Metropolitan Area Networks: Part 15.6: Wireless Body Area Networks. 2012. Available online: http://ieeexplore.ieee.org/servlet/opac?punumber 6161598 (accessed on 8 October 2017).

6. Chen, M.; Gonzalez, S.; Vasilakos, A.; Cao, H.; Leung, V. Body Area Networks: A Survey. Mob. Netw. Appl. 2010, 16, 171-193.

7. Fang, G.; Dutkiewicz, E.; Yu, K.; Vesilo, R.; Yu, Y. Distributed Inter-Network Interference Coordination for Wireless Body Area Networks. In Proceedings of the Global Telecommunications Conference (GLOBECOM 2010), Miami, FL, USA, 6-10 December 2010; pp. 1658-1686.

8. Dianati, M.; Shen, X.; Naikr, S. A New Fairness Index for Radio Resource Allocation in Wireless Networks. In Proceedings of the Wireless Communications and Networking Conference, New Orleans, LA, USA, 13-17 March 2005; pp. 712-717.

9. Amaldi, E.; Capone, A.; Malucelli, F.; Mannino, C. Optimization problems and models for planning cellular networks. In Handbook of Optimization in Telecommunications; Resende, M., Pardalos, P., Eds.; Springer: New York, NY, USA, 2006; pp. 917-939.

10. Kennington, J.; Olinick, E.; Rajan, D. Wireless Network Design: Optimization Models and Solution Procedures; Springer: New York, NY, USA, 2010.

11. Aardal, K.; Hoesel, S.; Koster, A.; Mannino, C.; Sassano, A. Models and solution techniques for frequency assignment problems. Ann. Oper. Res. 2007, 153, 79-129, doi:10.1007/s10479-007-0178-0. 
12. Olwal, T.O.; Masonta, M.T.; Mekura, F. Bio-inspired energy and channel management in distributed wireless multi-radio networks. IET Sci. 2014, 8, 380-390, doi:10.1049/iet-smt.2013.0204.

13. Dely, P.; D'Andreagiovanni, F.; Kassler, A. Fair optimization of mesh-connected WLAN hotspots. Wirel. Commun. Mob. Comput. 2015, 15, 924-946, doi:10.1002/wcm.2393.

14. Eisenblatter, A.; Geerdes, H. Capacity optimization for UMTS: Bounds and benchmarks for interference reduction. In Proceedings of the PIMRC, Cannes, France, 15-18 September 2008; pp. 1-6.

15. N-Sawaya, J.; Elhedhli, S. A nested benders decomposition approach for optimal W-CDMA telecommunication network planning. Nav. Res. Logist. 2010, 57, 519-539, doi:10.1002/nav.20419.

16. Mannino, C.; Rossi, F.; Smriglio, S. The Network Packing Problem in Terrestrial Broadcasting. Oper. Res. 2006, 54, 611-626, doi:10.1287/opre.1060.0312.

17. Rossi, F.; Sassano, A.; Smriglio, S. Models and Algorithms for Terrestrial Digital Broadcasting. Ann. Oper. Res. 2001, 107, 267-283, doi:10.1023/A:1014915519359.

18. Kim, E.; Youm, S.; Shon, T.; Kang, C. Asynchronous inter-network interference avoidance for wireless body area networks. J. Supercomput. 2013, 65, 562-579, doi:10.1007/s11227-012-0840-4.

19. Cheng, S.H.; Huang, C.Y. Coloring-based inter-WBAN scheduling for mobile wireless body area networks. IEEE Trans. Parallel Distrib. Syst. 2013, 24, 250-259, doi:10.1109/TPDS.2012.133.

20. Gu, F.; Liu, H.; Cheung, Y.M.; Xie, S. Optimal WCDMA network planning by multiobjective evolutionary algorithm with problem-specific genetic operation. Knowl. Inf. Syst. 2015, 45, 679-703, doi:10.1007/s10115-014-0799-y.

21. Gendron, B.; Scutella, M.G.; Garroppo, R.G.; Nencioni, G.; Tavanti, L. A branch-and-Benders-cut method for nonlinear power design in green wireless local area networks. Eur. J. Oper. Res. 2016, 255, 151-162, doi:10.1016/j.ejor.2016.04.058.

22. Kim, S.K.; Eom, D.S. Link-State-Estimation-Based Transmission Power Control in Wireless Body Area Networks. IEEE J. Biomed. Health Inf. 2013, 18, 1294-1302, doi:10.1109/JBHI.2013.2282864.

23. Gao, W.; Jiao, B.; Yang, G.; Hu, W.; Liu, J. Transmission power control for IEEE 802.15.6 body area networks. ETRI J. 2014, 36, 313-316, doi:10.4218/etrij.14.0213.0220.

24. Almeida, N.C. Beamforming and Power Control in Sensor Arrays Using Reinforcement Learning. Sensors 2015, 15, 6668-6687, doi:10.3390/s15030666.

25. D'Andreagiovanni, F. On Improving the Capacity of Solving Large-scale Wireless Network Design Problems by Genetic Algorithms. In Applications of Evolutionary Computation; Lecture Notes in Computer Science (LNCS 6625); Di Chio, C., Brabazon, A., Di Caro, G.A., Drechsler, R., Farooq, M., Grahl, J., Greenfield, G., Prins, C., Romero, J., Squillero, G., et al., Eds.; Springer: Basel, Switzerland, 2011; pp. 11-20.

26. Ahmed, T.; Moulec, Y. A QoS Optimization Approach in Cognitive Body Area Networks for Healthcare Applications. Sensors 2017, 17, 780, doi:10.3390/s17040780.

27. Xu, H.; Guo, C.; Zhang, L. Optimal Power Control in Wireless Powered Sensor Networks: A Dynamic Game-Based Approach. Sensors 2017, 17, 547, doi:10.3390/s17030547.

28. Tsouri, G.; Prieto, A.; Argade, N. On increasing network lifetime in body area networks using global routing with energy consumption balancing. Sensors 2012, 12, 13088-13108, doi:10.3390/s121013088.

29. Zhang, Y.; Zhang, B.; Zhang, S. A Lifetime Maximization Relay Selection Scheme in Wireless Body Area Networks. Sensors 2017, 17, 1276, doi:10.3390/s17061267.

30. Bauschert, T.; Busing, C.; D'Andreagiovanni, F.; Koster, A.; Kutschka, M.; Steglich, U. Network planning under demand uncertainty with robust optimization. IEEE Commun. Mag. 2014, 52, 178-185, doi:10.1109/MCOM.2014.6736760.

31. Garroppo, R.; Nencioni, G.; Scutella, M.G.; Tavanti, L. Robust optimisation of green wireless LANs under rate uncertainty and user mobility. Electron. Notes Discrete Math. 2016, 52, 221-228, doi:10.1016/j.endm.2016.03.029.

32. D'Andreagiovanni, F.; Nardin, A.; Natalizio, E. A Fast ILP-Based Heuristic for the Robust Design of Body Wireless Sensor Networks. In EvoApplications 2017: Applications of Evolutionary Computation; Springer Lecture Notes in Computer Science (LNCS 10199); Squillero, G., Sim, K., Eds.; Springer: Basel, Switzerland, 2017; pp. 1-17.

33. D'Andreagiovanni, F.; Nardin, A. Towards the fast and robust optimal design of wireless body area networks. Appl. Soft Comput. 2015, 37, 971-982, doi:10.1016/j.asoc.2015.04.037. 
34. D'Andreagiovanni, F. Revisiting wireless network jamming by SIR-based considerations and multiband robust optimization. Optim. Lett. 2015, 9, 1495-1510, doi:10.1007/s11590-014-0839-2.

35. Heikkinen, T.; Prekopa, A. Optimal power control in a wireless network using stochastic link coefficients. Nav. Res. Logist. 2004, 52, 178-192, doi:10.1002/nav.20054.

36. Olinick, E.; Rosenberger, J. Optimizing revenue in CDMA networks under demand uncertainty. Eur. J. Oper. Res. 2008, 186, 812-825, doi:10.1016/j.ejor.2007.02.048.

37. Zakrzewska, A.; D’Andreagiovanni, F.; Ruepp, S.; Berger, M.S. Biobjective Optimization of Radio Access Technology Selection and Resource Allocation in Heterogeneous Wireless Networks. In Proceedings of the 9th International Workshop on Resource Allocation, Cooperation and Competition in Wireless Networks, Tsukuba Science City, Japan, 13-17 May 2013; pp. 652-658.

38. Chen, X.; Xu, Y.; Liu, A. Cross Layer Design for Optimizing Transmission Reliability, Energy Efficiency, and Lifetime in Body Sensor Networks. Sensors 2017, 17, 900, doi:10.3390/s17040900.

39. Yao, X.W.; Wang, W.L.; Yang, S.H.; Cen, Y.F. Bio-inspired self-adaptive rate control for multi-priority data transmission over WLANs. IEEE Comput. Commun. 2014, 53, 73-83, doi:10.1016/j.comcom.2014.07.010.

40. Cucker, F.; Smale, S. Emergent behavior in flocks. IEEE Trans. Autom. Control 2007, 52, 852-862, doi:10.1109/TAC.2007.895842.

41. Dong, J.; Smith, D. Joint Relay Selection and Transmit Power Control for Wireless Body Area Networks Coexistence. In Proceedings of the IEEE International Conference on Communications (ICC), Sydney, Australia, 10-14 June 2014; pp. 5676-5681.

42. Kazemi, R.; Vesilo, R.; Dutkiewicz, E.; Fang, G. Inter-Network Interference Mitigation in Wireless Body Area Networks Using Power Control Games. In Proceedings of the International Symposium on Communications and Information Technologies (ISCIT), Tokyo, Japan, 26-29 October 2010; pp. 81-86.

43. Zhang, Z.; Wang, H.; Wang, C.; Fang, H. Interference Mitigation for Cyber-Physical Wireless Body Area Network System Using Social Networks. IEEE Trans. Emerg. Top. Comput. 2013, 1, 121-132, doi:10.1109/TETC.2013.2274430.

44. Quwalder, M.; Rao, J.; Blswas, S. Body-Posture-Based Dynamic Link Power Control in Wearable Sensor Networks. IEEE Commun. Mag. 2010, 48, 134-142, doi:10.1109/MCOM.2010.5496890.

45. Suzuki, K.; Nishiyama, H.; Kato, N.; Ujikawa, H.; Suzuki, K.; Yoshimoto, N. A bandwidth allocation method to improve user QoS satisfaction without decreasing system throughput in wireless access networks. In Proceedings of the IEEE 23rd International Symposium on Personal, Indoor and Mobile Radio Communications (PIMRC), Sydney, Australia, 9-12 September 2012; pp. 1430-1435.

46. Cavallari, R.; Martelli, F.; Rosini, R.; Buratti, C.; Verdone, R. A survey on wireless body area networks: Technologies and design challenges. IEEE Commun. Surv. Tutor. 2014, 16, 1635-1657, doi:10.1109/SURV.2014.012214.00007.

47. Lewis, D. 802.15.6 Call for Applications-Response Summary. 2008. Available online: https: / mentor.ieee.org/802.15/dcn/08/15-08-0407-06-0006-tg6-applications-summary.doc (accessed on 8 October 2017).

48. Yazdandoost, K.Y.; Sayrafian-Pour, K. Channel Model for Body Area Network (BAN). 2009. Available online: https: / www.nist.gov/ sites/default/ files/documents /2017/05/09/15-08-0780-09-0006tg6-channel-model.pdf (accessed on 8 October 2017).

49. Jain, R.; Chiu, D.M.; Hawe, W. A Quantitative Measure of Fairness and Discrimination for Resource Allocation in Shared Computer Systems. DEC Research Report TR-301. 1984. Available online: http: / / www1.cse.wustl.edu/ jain/papers/ftp/fairness.pdf (accessed on 8 October 2017).

(c) 2017 by the authors. Licensee MDPI, Basel, Switzerland. This article is an open access article distributed under the terms and conditions of the Creative Commons Attribution (CC BY) license (http://creativecommons.org/licenses/by/4.0/). 